\title{
Randomized incremental construction of abstract Voronoi diagrams*
}

\author{
Rolf Klein \\ Fern Universität Hagen, Praktische Informatik VI, W-5800 Hagen, Germany \\ Kurt Mehlhorn and Stefan Meiser \\ Max-Planck-Institut für Informatik, W-6600 Saarbrücken, Germany \\ Communicated by Kenneth Clarkson \\ Submitted 18 June 1991 \\ Accepted 26 March 1993
}

\begin{abstract}
Abstract Voronoi diagrams were introduced by R. Klein (1988) as an axiomatic basis of Voronoi diagrams. We show how to construct abstract Voronoi diagrams in time $\mathrm{O}(n \log n)$ by a randomized algorithm, which is based on Clarkson and Shor's randomized incremental construction technique (1989). The new algorithm has the following advantages over previous algorithms:

- It can handle a much wider class of abstract Voronoi diagrams than the algorithms presented in by Klein (1989) and, Mehlhorn, Meiser and O'Dúnlaing (1991).

- It can be adapted to a concrete kind of Voronoi diagram by providing a single basic operation, namely the construction of a Voronoi diagram of five sites. Moreover, all geometric decisions are confined to the basic operation, and using this operation, abstract Voronoi diagrams can be constructed in a purely combinatorial manner.
\end{abstract}

\section{Introduction}

The Voronoi diagram of a set of sites in the plane partitions the plane into regions, called Voronoi regions, one to a site. The Voronoi region of a site $s$ is the set of points in the plane for which $s$ is the closest site among all the sites.

Correspondence to: Rolf Klein, Fern Universität Hagen, Praktische Informatik VI, W-5800 Hagen, Germany.

* A preliminary version of this paper was presented at the SIGAL Symposium on Algorithms, Tokyo 1990. This work was supported in part by the ESPRIT Basic Research Actions Program of the EC under contract No. 7141 (ALCOM II), by the BMFT (Förderungskennzeichen ITS 9103), and by the DFG grant $\mathrm{Kl} 655 / 2-1$. 
The Voronoi diagram has many applications in diverse fields, cf. Leven and Sharir [18] or Aurenhammer [2] for a list of applications and a history of Voronoi diagrams. Different types of diagrams result from considering different notions of distance, e.g., Euclidean or $L_{p}$-norm or convex distance functions, and different sorts of sites, e.g., points, line segments, or circles. For many types of diagrams efficient construction algorithms have been found, which are either based on the divide-and-conquer technique due to Shamos and Hoey [21], the sweepline technique due to Fortune [10], geometric transforms due to Brown [6] and Edelsbrunner and Seidel [9], or the randomized incremental construction technique due to Clarkson and Shor [9].

A unifying approach to Voronoi diagrams has been proposed by Klein [13-16], cf. [9] for a related approach. He does not use the concept of distance as the basic notion but rather the concept of bisecting curves, i.e., he assumes for each pair $\{p, q\}$ of sites the existence of a bisector $J(p, q)$, which is homeomorphic to a line and divides the plane into a $p$-region and a $q$-region. The intersection of all $p$-regions for different $q$ 's is then the Voronoi region of site $p$. He also postulates that Voronoi regions are simply-connected and partition the plane. He shows that abstract Voronoi diagrams already have many of the properties of concrete Voronoi diagrams, cf. Section 2.

At present there are two algorithms for the construction of abstract Voronoi diagrams. Both algorithms assume that certain elementary operations on bisecting curves, e.g., computation of the intersections, take $O(1)$ time, and both algorithms can handle only subclasses of abstract Voronoi diagrams.

Klein [16] presented an off-spring of the Shamos and Hoey divide-and-conquer algorithm. He has to assume that any set $S$ of sites can be split in time $\mathrm{O}(|S|)$ into approximately equal sized subsets $L$ and $R$ such that the bisector between $L$ and $R$ (= the common boundary of regions in $l$, with regions in $R$ ) is acyclic and, under this assumption, constructs the Voronoi diagrams of $n$ sites in time $\mathrm{O}(n \log n)$. There are cases, e.g., points with additive weights in the Euclidean plane, where it is not known if such partitions exist.

Mehlhorn, Meiser and Ó'Dúnlaing [19] have presented an off-spring of the Clarkson and Shor randomized incremental algorithm. They have to assume that the set of bisectors is regular, i.e., no four of them share a point and any point of intersection of two bisectors is a proper crossing of the bisectors. Under these assumptions, their algorithm runs in expected time $\mathrm{O}(n \log n)$, the average being taken over all permutations of the input. There are cases, e.g., point sites in the Manhattan metric, where this assumption does not hold.

In this paper, we extend the randomized incremental algorithm and show that it can handle abstract Voronoi diagrams in (almost) their full generality, cf. the remark following Definition 1 in Section 2 for the minor restriction which we have to make. The algorithm runs in expected time $\mathrm{O}(n \log n)$ and is as simple as the algorithm in [19]. However, its correctness proof and running time analysis are more involved. The algorithm is uniform in the sense that only a single operation, 
namely the construction of a Voronoi diagram for 5 sites, depends on the specific type of Voronoi diagram and has to be programmed in order to adapt the algorithm to the type of the diagram. Moreover, all numerical operations take place within this particular operation.

In particular, comparisons only take place between objects which are related in the topology of the diagram. The incremental algorithm of Guibas and Stolfi [12] for Euclidean diagrams also has this property but neither the Plane-Sweep- nor the Divide-and-Conquer-algorithm do. Both algorithms need to sort the sites by $x$-coordinates. Moreover, the Plane-Sweep-algorithm sorts the computed events by $x$-coordinates; the Divide-and-Conquer-algorithm sorts the nodes of the diagram by $y$-coordinates in its merge step. In both cases, objects that are not at all related in their topology are compared to each other. Therefore, it may be difficult to make geometric decisions in a consistent manner. From a programmer's point of view, concentrating the numerical computations inside a single operation may facilitate the handling of approximate arithmetic. We want to emphasize that the fact that our basic operation operates on five sites does not imply that an implementation of the basic operation must use tests which involve five sites and therefore are likely to have high algebraic degree. We show in Section 6 that four sites suffice for simple families of bisectors, i.e., families of bisectors where the Voronoi diagram of any three sites has at most one vertex.

As mentioned above, our algorithm is based on Clarkson and Shor's randomized incremental construction technique [8]. We make use of the refinement proposed in $[11,3,5,4]$; in particular, we use the notion of history graph instead of the original conflict graph.

An earlier version of the algorithm, which uses a conflict graph instead of a history graph, was implemented by Zimmer [22]. We have used it to construct Powerdiagrams, Voronoi diagrams of line segments under the Euclidean metric, and Voronoi diagrams of points under both the Euclidean and the $L_{1}$-metric. The general, diagram-independent part of the algorithm thereby comprises circa 2700 lines of code. This should be compared to the amount of code needed to implement the diagram-specific part of the algorithm (basic operation and drawing routines). This part varies between 450 lines for points under the Euclidean metric and 3250 lines of code for line segment sites under the Euclidean metric. For Powerdiagrams and diagrams of points under the $L_{1}$-metric we needed 550 and 850 lines, respectively. Note that approximately one sixth of this is code for drawing the diagram on the screen.

The present paper is not quite in line with a popular trend in computational geometry: to use symbolic perturbation to establish general position, then to use an algorithm which can only handle inputs in general position (e.g., the algorithm of [19]), and finally to produce the true output by a limit process (essentially by shrinking some edges and collapsing vertices). We cannot follow this approach for several reasons. Firstly, there is no efficient perturbation technique available for abstract Voronoi diagrams. Klein [15] showed that any admissible family of 

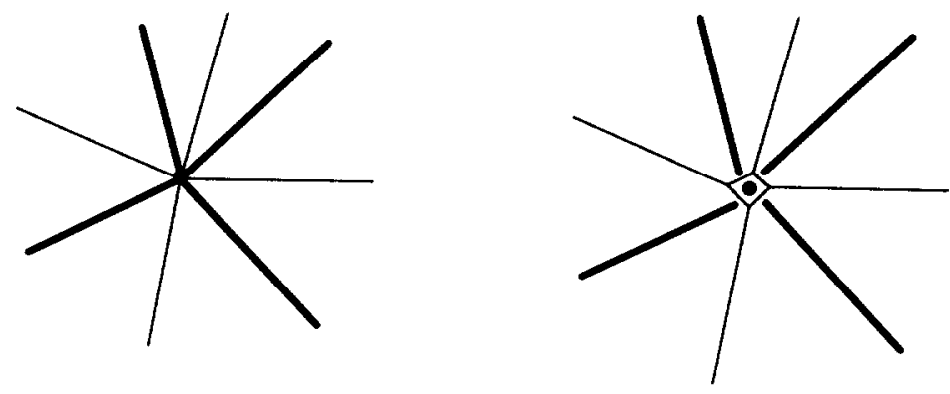

Fig. 1. The Voronoi diagram for a degenerate and a perturbed input.

bisectors can be perturbed to general position, but his perturbation technique can require exponential time and needs to know the Voronoi diagram. Secondly, we did not want to use a perturbation technique which is outside our algorithm, e.g., one which uses properties of the particular kind of diagram under construction, because this would require programming the limit process for each particular kind of diagram. We believe that it is better to make the algorithm as uniform as possible and to confine the dependency on the particular kind of digram to a single subroutine (here, the construction of a five sites diagram). Thirdly, perturbation and limit process are not always a trivial task. Consider for example the Voronoi diagram of a point and four open line segments touching in this point, cf. Fig. 1. The perturbation is nontrivial, since it should not introduce intersections between the segments. The limit process is nontrivial, since it must collapse the point with the four endpoints of the segments. But these features are not directly linked in a typical data structure for the perturbed diagram. Finally, perturbation might increase the running time by more than a constant factor. The expected running time of one incremental step of our algorithm is proportional to

$$
\sum_{i \leqslant n} \frac{f_{i}}{i^{2}}(n-i)
$$

where $f_{i}$ is the expected number of edges in a diagram for a random subset of $i$ sites from the $n$ given sites. Since regions in abstract Voronoi diagrams may be empty, we may have $f_{i}=o(i)$. In such a situation, the running time of the algorithm can be $\mathrm{o}(n \log n)$. Perturbation creates general position and may increase $f_{i}$ to $\Theta(i)$. Finally, we believe that despite the handling of degenerate cases the algorithm presented in this paper is still very simple. Degenerate cases complicate the discussion of correctness and running time, but affect the algorithm itself only to a small extent.

The paper is organized as follows: In Section 2 we introduce abstract Voronoi diagrams; we give the relevant definitions and state some properties. In Section 3 we investigate the Voronoi diagram of five sites and present the basic operation of our algorithm. The algorithm is then given in Section 4. Section 5 contains the 
analysis of the algorithm's running time and space requirements. In Section 6 we inspect the basic operation for a subclass of abstract Voronoi diagrams in more detail.

Throughout the paper, we use the following notation: For a subset $X \subseteq \mathbb{R}^{2}$ the closure, boundary and interior of $X$ are denoted by $\bar{X}$, bd $X$ and int $X$, respectively.

\section{Abstract Voronoi diagrams}

Let $n \in \mathbb{N}$, and for every pair of integers $p, q$ such that $1 \leqslant p \neq q<n$ let $D(p, q)$ be either empty or an open unbounded subset of $\mathbb{R}^{2}$ and let $J(p, q)$ be the boundary of $D(p, q)$. We postulate:

(1) $J(p, q)=J(q, p)$ and for each $p, q$ such that $p \neq q$ the regions $D(p, q)$, $J(p, q)$ and $D(q, p)$ form a partition of $\mathbb{R}^{2}$ into three disjoint sets.

(2) If $\emptyset \neq D(p, q) \neq \mathbb{R}^{2}$ then $J(p, q)$ is homeomorphic to the open interval $(0,1)$.

We call $J(p, q)$ the bisecting curve for sites $p$ and $q$ and $D(p, q)$ the region of dominance of $p$ over $q$. Following [16], the abstract Voronoi diagram is now defined as follows.

Definition 1. Let $S=\{1, \ldots, n-1\}$ and

$$
\begin{aligned}
& R(p, q):= \begin{cases}D(p, q) \cup J(p, q) & \text { if } p<q, \\
D(p, q) & \text { if } p>q,\end{cases} \\
& \operatorname{EVR}(p, S):=\bigcap_{q \in S, q \neq p} R(p, q), \\
& \operatorname{VR}(p, S):=\operatorname{int} \operatorname{EVR}(p, S), \\
& \operatorname{V(S)}:=\bigcup_{p \in S} \operatorname{bd} \operatorname{EVR}(p, S),
\end{aligned}
$$

$\operatorname{VR}(p, S)$ is called the Voronoi region of $p$ or $p$-region w.r.t. $\mathrm{S}, \operatorname{EVR}(p, S)$ is called the extended Voronoi region of $p$ w.r.t. $S$, and $\mathrm{V}(S)$ is called the Vorono diagram of $S$. The elements of $S$ are referred to as sites.

We require the Voronoi regions and the bisecting curves to satisfy the following two conditions:

(3) Any two bisecting curves intersect in only a finite number of connected components.

(4) For all non-empty subset $S^{\prime}$ of $S$

(a) for all $p \in S^{\prime}$ for which $\operatorname{EVR}\left(p, S^{\prime}\right)$ is non-empty: $\operatorname{VR}\left(p, S^{\prime}\right)$ is non-empty and $\operatorname{EVR}\left(p, S^{\prime}\right)$ and $\operatorname{VR}\left(p, S^{\prime}\right)$ are path-connected,

(b) $\mathbb{R}^{2}=\bigcup_{p \in S^{\prime}} \operatorname{EVR}\left(p, S^{\prime}\right)$. 

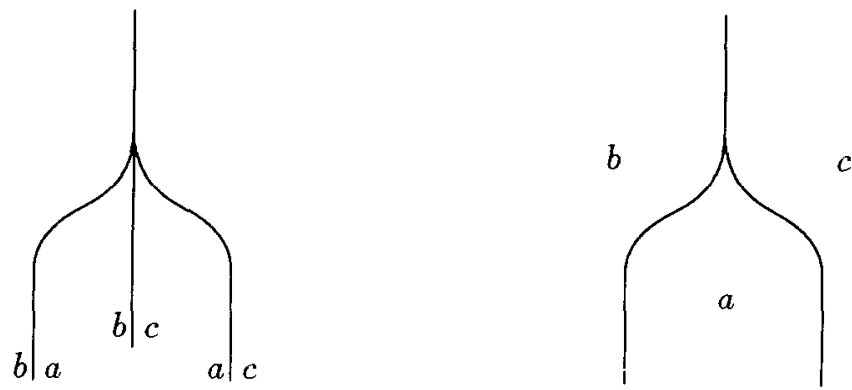

Fig. 2. A family of three bisectors for sites $a, b$ and $c$, and the induced Voronoi diagram. For each bisector the two sites separated by the bisector are indicated near to the bisector.

Abstract Voronoi diagrams include a large number of concrete Voronoi diagrams, e.g., Voronoi diagrams for point sites under any $L_{p}$-metric, $1 \leqslant p \leqslant \infty$, or under any convex distance function, whose unit circle is semi-algebraic. They furthermore comprise Power-diagrams, and Voronoi diagrams for line segments or circles under the Euclidean metric. The line segments may cven touch at their endpoints, thus possibly forming polygons, and the circles are allowed to intersect. Voronoi diagrams for disjoint convex figures under a convex distance function are also included, provided their bisectors satisfy our Condition 3. Of course, there are also negative examples; Euclidean Voronoi diagrams for point sets with multiplicative weights or Euclidean Voronoi diagrams for nonconvex figures, e.g., circular arcs. In both cases there may be circular bisecting curves violating our Condition 2. Figs. 2 and 3 show two abstract Voronoi diagrams.

Abstract Voronoi diagrams are defined by means of bisecting curves. Depending on the concrete Voronoi diagram, the complexity of the bisectors may vary considerably. For the sake of simplicity we assume however that bisectors are computationally simple (see Section 3 ). We will show that under these assumptions abstract Voronoi diagrams can be constructed in time $\mathrm{O}(n \log n)$ by a randomized algorithm.
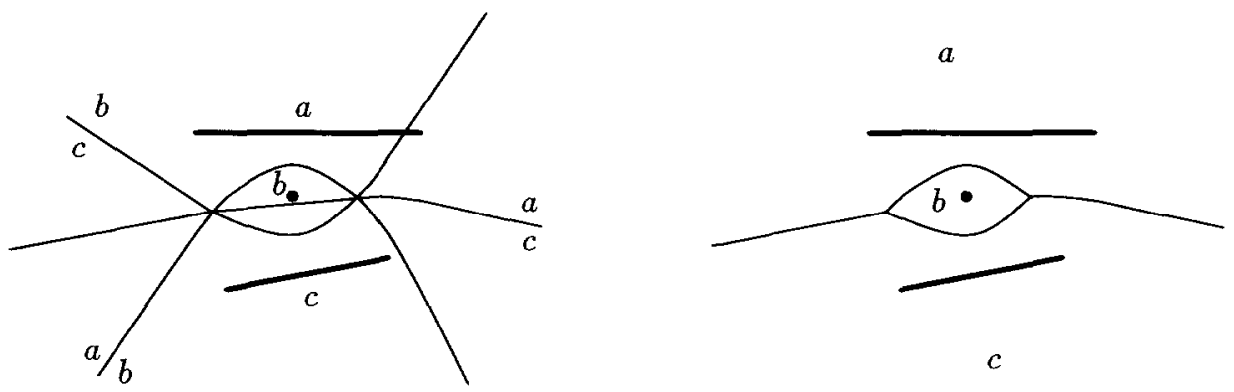

Fig. 3. A family of three bisectors arising from two line segment sites $a$ and $c$ and one point site $b$ under the Euclidean metric. The bisectors are drawn as thin curves, the segments are fat. 


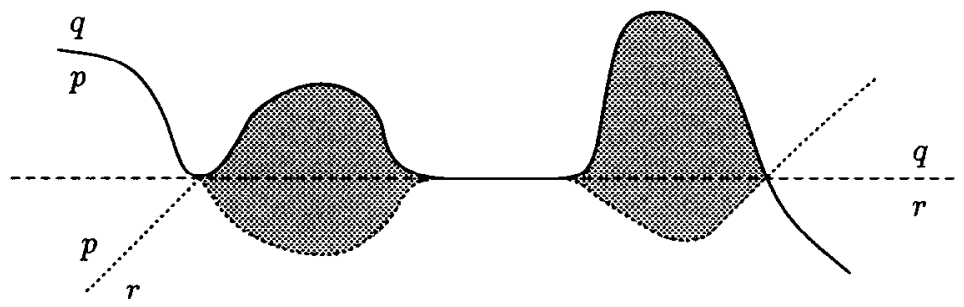

Fig. 4. A degenerate case.

Remarks. (1) In [16] Condition $4 \mathrm{~b}$ is shown to be equivalent to the following transitivity property: $R(p, q) \cap R(q, r) \subseteq R(p, r)$ for any three pairwise distinct sites $p, q, r \in S$.

(2) The union in $4 \mathrm{~b}$ is disjoint by the definition of Voronoi regions.

(3) Our Definition of an abstract Voronoi diagram differs in two respects from Klein's original definition in [16]. Firstly, we also allow empty Voronoi regions which does not harm Klein's theory. Secondly, our Condition 4a is slightly more restrictive than the one in [16]. There, only the extended Voronoi regions are required to be path-connected, but not their interior. Fig. 4 shows a system of bisectors for three sites $p, q, r$ which satisfies Klein's assumptions if $p<q$ and $p<r$. Our assumptions, however, exclude this example since its $p$-regions is disconnected.

Definition 2. An edge $e$ of $\mathrm{V}(S)$ is a maximal connected subset of $\mathrm{V}(S)$ such that every point $x \in e$ lies on bd $\operatorname{VR}(p, S)$ for exactly two sites $p$ of $S$. The edge is said to separate the regions of these two sites. A vertex $v$ of $\mathrm{V}(S)$ is a point $x \in \mathrm{V}(S)$ which lies on bd $\operatorname{VR}(p, S)$ for at least three sites $p$ of $S$.

Fact 1 (piece of pie fact). (1) All but finitely many points of $\mathrm{V}(S)$ belong to an edge of $\mathrm{V}(S)$.

(2) For each point $x \in \mathrm{V}(S)$ there are arbitrarily small neighborhoods $U$ of $x$ having the following properties: $\mathrm{V}(S) \cap \mathrm{bd} U$ consists of finitely many points. Let $w_{1}, \ldots, w_{h}$ denote these points as encountered in a clockwise traversal of bd $U$. Then $h \geqslant 2$ and $\mathrm{V}(S) \cap U$ is the union of curve segments $\beta_{1}, \ldots, \beta_{h}$ where $\beta_{i}$ connects $x$ to $w_{i}$ and the $\beta_{i}$ 's are disjoint except at their common endpoint $x$. For each $i, 1 \leqslant i \leqslant h$, there is a site $p_{i} \in S$ such that the open 'piece of pie' bordered by $\beta_{i}, \beta_{i+1}($ read indices $\bmod h)$ is contained in $\operatorname{VR}\left(p_{i}, S\right)$. Then $p_{i} \neq p_{j}$ for $i \neq j$. For each $\beta_{i}$ there is a site $q_{i} \in S$, such that $\beta_{i}-x \subseteq \operatorname{EVR}\left(q_{i}, S\right)$. We have $q_{i} \leqslant$ $\min \left\{p_{i-1}, p_{i}\right\}$. The point $x$ belongs to $\operatorname{EVR}(p, S)$, where $p=$ $\min \left\{p_{1}, \ldots, p_{h}, q_{1}, \ldots, q_{h}\right\}$. Also, only the extended Voronoi region of site $p$ can be encountered more than once on the march around bd $U$.

Fig. 5 illustrates Fact 1. Fact 1 is an immediate derivative of Theorem 2.3.5 of [16]. There only $p_{i-1} \neq p_{i}$ was claimed. The claim $p_{i} \neq p_{j}$ for $i \neq j$ made here follows from our strengthened Condition 4a and Lemma 2.2.4 of [16]. 


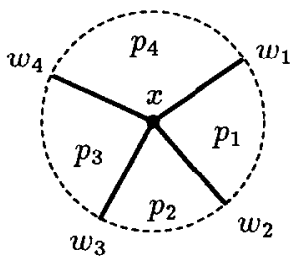

a vertex $x$

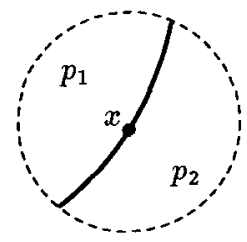

a point $x$ on an edge $x \in E V R\left(\min \left\{p_{1}, p_{2}\right\}, S\right)$

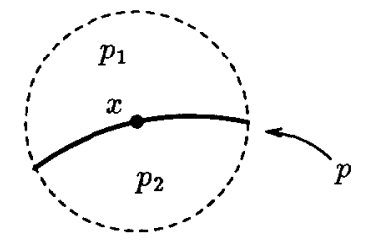

$x \in E V R(p, S)$ and

$p<\min \left\{p_{1}, p_{2}\right\}$

Fig. 5. Illustration of Fact 1.

For the sequel, it is helpful to restrict attention to the 'finite part' of $\mathrm{V}(S)$. Let $\Gamma$ be a simple closed curve such that in the outer domain of $\Gamma$ the curve segments of any two bisectors are either disjoint or identical. We add a site $\infty$ to $S$, define $J(p, \infty)=J(\infty, p)=\Gamma$ for all $p, 1 \leqslant p<n$, and $D(\infty, p)$ to be the outer domain of $\Gamma$ for each $p, 1 \leqslant p<n$.

Fact 2. $\mathrm{V}(S)$ is connected. The extended Voronoi region of a site $p \in S-\{\infty\}$ is simply-connected, each non-empty Voronoi region $\operatorname{VR}(p, S), p \in S-\{\infty\}$, is homeomorphic to an open disc and its boundary is a simple closed curve. The Voronoi region of site $\infty$ is not simply connected but it has only one hole being the inner domain of $\Gamma$. A Voronoi diagram can be represented as a planar graph in a Inatural way. The vertices and edges of the graph are the vertices and edges of $\mathrm{V}(S)$, respectively; the faces of the graph correspond to the non-empty Vorono regions. We use $\mathrm{V}(S)$ also to denote this graph.

For a proof of Fact 2 see Lemma 2.2.4 and Theorems 2.3.5 and 2.5.5 [16].

The extended Voronoi region $\operatorname{EVR}(p, S)$, for a site $p \in S-\{\infty\}$, consists of its Voronoi region, some vertices and edges on the boundary of $\operatorname{VR}(p, S)$, and some other vertices and edges of $\mathrm{V}(S)$. The other edges and vertices form trees rooted at $\operatorname{bd} \operatorname{VR}(p, S)$, cf. Fig. 6.

We will next return to the example in Fig. 2 in order to illustrate the concepts

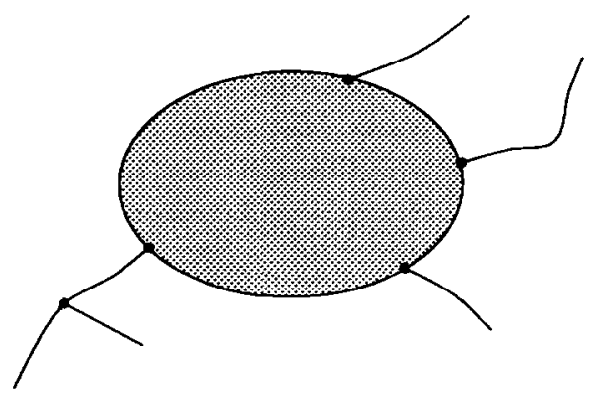

Fig. 6. An extended Voronoi region $\operatorname{EVR}(p, S)$. 


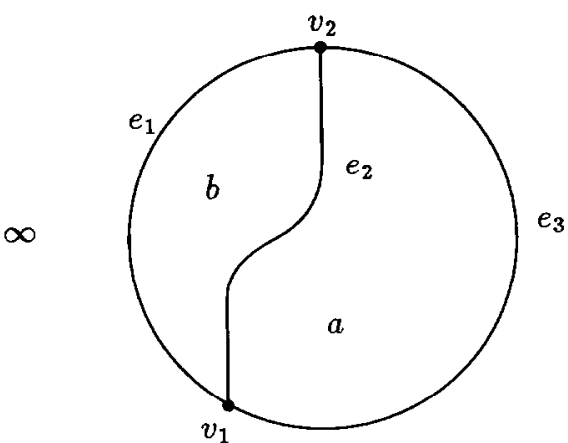

$$
V(\{a, b, \infty\})
$$

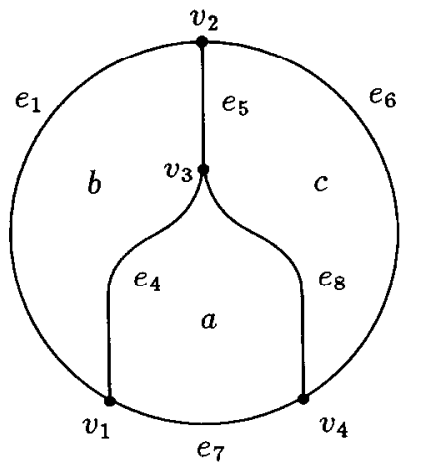

$$
V(\{a, b, c, \infty\})
$$

$\infty$

Fig. 7. The enclosing circle represents $\Gamma$. Edges $e_{1}$ and $e_{3}$ are part of $J(b, \infty)=\Gamma$ and $J(a, \infty)=\Gamma$, respectively.

introduced so far. We will use this example as our running example throughout the paper.

Example. Fig. 7 shows $V(\{a, b, \infty\})$ and $V(\{a, b, c, \infty\})$ for the bisectors defined in Fig. 2. Assume $a<b<c<\infty$. Then edges $e_{2}$ and $e_{3}$ belong to $\operatorname{EVR}(a,\{a, b, \infty\})$ and edges $e_{4}, e_{5}, e_{7}$ and $e_{8}$ belong to $\operatorname{EVR}(a,\{a, b, c, \infty\})$.

\section{Inserting a new site}

The algorithm presented in Section 4 constructs the Voronoi diagram $\mathrm{V}(S)$ by adding one site after the other. In this section we investigate the part of a Voronoi diagram that is 'cut off' by the insertion of a new site. For the remainder of the section, let $R \subseteq S, \infty \in R, s \in S-R, \mathscr{S}=\mathrm{VR}(s, R \cup\{s\})$, and $\mathscr{E}=\mathrm{V}(R) \cap$ $\overline{\mathscr{F}}$. Then, according to Fact 2 , bd $\mathscr{S}=$ bd $\overline{\mathscr{F}}$ is a simple closed curve.

Lemma 1. If $\mathscr{S} \neq \emptyset$ then $\mathscr{E}$ is a non-empty connected set which intersects bd $\mathscr{S}$. Moreover, $\mathscr{E}$ is not just a single point.

Proof. If $\mathscr{E}$ were empty, then $\overline{\mathscr{T}} \subseteq \operatorname{VR}(p, R)$ for some $p \in R-\{\infty\}$. Consequently, $\operatorname{VR}(p, R \cup\{s\})$ would not be simply connected. Now let $\mathscr{E}_{1}, \mathscr{E}_{2}, \ldots, \mathscr{E}_{k}$ be the connected components of $\mathscr{E}$ for some $k$. Observe that no $\mathscr{E}_{j}$ can be entirely contained inside $\mathscr{S}$ because otherwise $\mathrm{V}(R)$ would not be connected, a contradiction.

Assume $k \geqslant 2$. Then a path $\mathscr{P} \subseteq \overline{\mathscr{T}}-\mathscr{E}$ exists, connecting two points $x$ and $y$ on the boundary of $\mathscr{S}$ and separating $\mathscr{E}_{1}$ from $\mathscr{E}_{2}$, cf. Fig. 8. From $\mathscr{P} \cap \mathscr{E}=\emptyset$ we have $\mathscr{P} \cap \mathrm{V}(R)=\emptyset$ and thus $\mathscr{P} \subseteq \mathrm{VR}(r, R)$ for a site $r \in R-\{\infty\} . x, y \in \mathscr{P}$ implies that all sufficiently small neighborhoods $U(x)$ and $U(y)$ are entirely contained in 


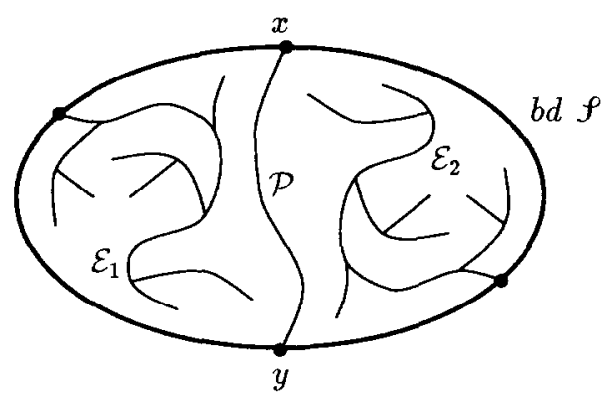

Fig. 8. Path $\mathscr{P}$ and two connected components of $\mathscr{E}$.

$\operatorname{VR}(r, R)$. The points in the intersection of these neighborhoods with the complement of $\overline{\mathscr{S}}$ thus lie in $\operatorname{VR}(r, R \cup\{s\})$ and can be connected by a path $\mathscr{Q} \subseteq \mathrm{VR}(r, R \cup\{s\}) \subseteq \mathrm{VR}(r, R)$. The cycle $\mathscr{P} \circ \mathscr{Q}$ is therefore entirely contained in $\operatorname{VR}(r, R)$ and contains $\mathscr{E}_{1}$ or $\mathscr{E}_{2}$ in its interior. This is a contradiction.

At this point we have shown that $\mathscr{E}$ is a non-empty connected set which intersects bd $\mathscr{f}$. Assume now that $\mathscr{E}$ is a single point. This point, say $v$, is either a vertex of $\mathrm{V}(R)$ or lies on an edge of $\mathrm{V}(R)$. In either case, one of the regions of $\mathrm{V}(R)$ incident to $v$ in $\mathrm{V}(R)$ is split by $\mathscr{S}$ in a neighborhood of $v$ and hence represented twice at $v$ in $\mathrm{V}(R \cup\{s\})$, cf. Fig. 9, a contradiction to the piece of pie fact.

Note that Lemma 1 implies in particular, that if $\mathscr{S} \neq \emptyset$, then $\overline{\mathscr{S}}$ intersects an edge of $\mathrm{V}(R)$. Lemma 2 discusses the various forms which an intersection between $\overline{\mathscr{S}}$ and an edge $e$ of $\mathrm{V}(R)$ can have.

Lemma 2. Let $e$ be an edge of $\mathrm{V}(R)$. If $e \cap \overline{\mathscr{S}} \neq \emptyset$, then either $e \cap \overline{\mathscr{S}}=\mathrm{V}(R) \cap \overline{\mathscr{S}}$ and $e \cap \overline{\mathscr{S}}$ is a single component or $e-\overline{\mathscr{S}}$ is a single component (possibly empty).

Proof. Assume first that $e \cap \overline{\mathscr{S}}=\mathrm{V}(R) \cap \overline{\mathscr{S}}$. Since $\mathrm{V}(R) \cap \overline{\mathscr{S}}$ is connected according to Lemma $1, e \cap \overline{\mathscr{S}}$ is also connected. Assume next that $e \cap \overline{\mathscr{S}} \neq \mathrm{V}(R) \cap \overline{\mathscr{S}}$. Then for every point $x \in e \cap \overline{\mathscr{S}}$ one of the subpaths of $e$ connecting $x$ to an endpoint of $e$ must be contained in $\overline{\mathscr{S}}$, since $\mathrm{V}(R) \cap \mathscr{S}$ is a connected set. Hence $e-\overline{\mathscr{S}}$ is a single component.
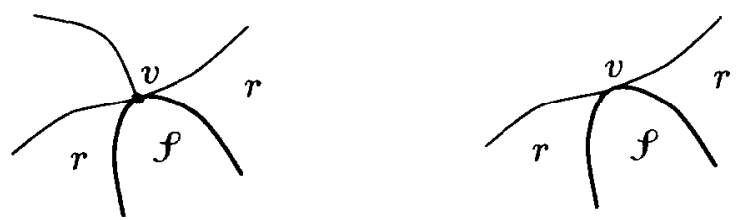

Fig. 9. $r$-region is represented twice at $v$, a contradiction to Fact 1. 


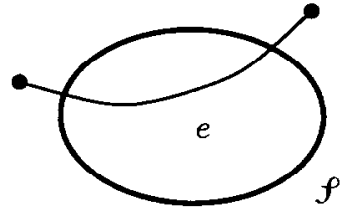

(a)

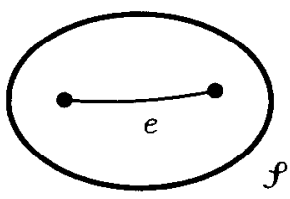

(b)

allowed

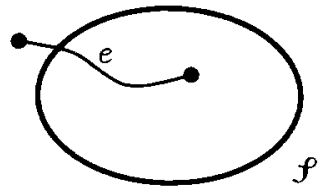

(c)

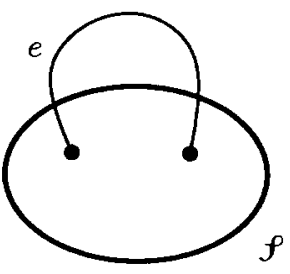

(d)

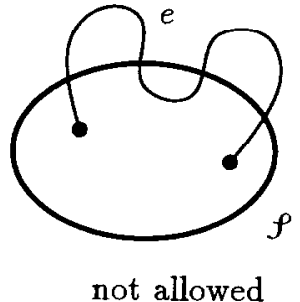

not allowed

Fig. 10. Four situations as allowed by Lemma 2 and an impossible one.

Fig. 10 shows some possible and impossible configurations for $e \cap \overline{\mathscr{Y}}$ according to Lemma 2. Fig. 11, which also illustrates the following Definition 3, shows that cases (a) and (d) of Fig. 10 arise even for Euclidean diagrams of line segment sites.

We close this section with some notations that we need in the forthcoming sections.

Definition 3. Let $e$ be an edge of $\mathrm{V}(R)$ and let $v$ be an endpoint of $e$. Then:

(1) $s$ intersects $e$ with respect to $R$ iff $e \cap \overline{\mathscr{S}} \neq \emptyset$.

(2) $s$ clips $e$ at $v$ with respect to $R$ iff $e \cap \overline{\mathscr{S}}$ contains a component incident to $v$.

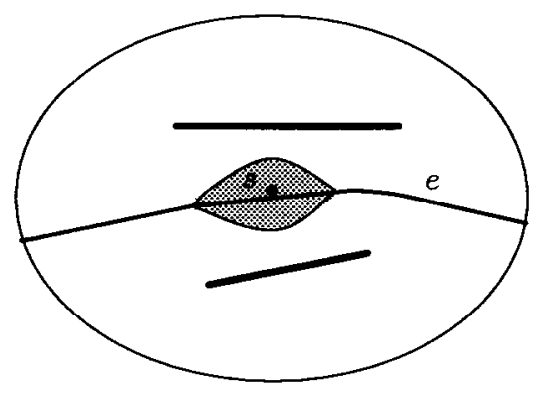

site $s$ intersects edge $e$ without clipping

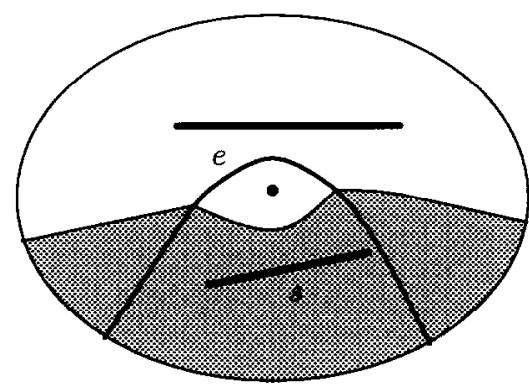

site $s$ clips $e$ at both endpoints

Fig. 11. The Euclidean Voronoi diagram of two line segment sites and one point site. Two sites form an edge $e$. The third site $s$ is inserted into the diagram of the other two sites and thereby intersects edge $e$. The Voronoi region of site $s$ is shown shaded. 


\section{The basic operation}

Computing the intersection between an edge and the region of a new site is the fundamental operation in our algorithm. We have already seen in Lemma 2 that there are only a few types of such intersections. In this section we show that a particular type of intersection can be extracted from the Voronoi diagram of only five sites and therefore computed in constant time. The five sites involved are the newly added site and four sites 'defining' the edge. We first specify how sites 'define' edges. As above let $R \subseteq S$ and $\infty \in R$.

Definition 4. Let $p, q, r$ and $t$ be sites in $R$.

(1) A vertex $v$ of $\mathrm{V}(R)$ is called a $p q r$-vertex (see Fig. 12), if $v$ is incident to the $p-, q-$, and $r$-regions, and there is a clockwise traversal of the regions incident to $v$ which encounters $p$-region before $q$-region before $r$-region before $p$-region.

(2) An edge $e$ of $\mathrm{V}(R)$ is called a prqt-edge (see Fig. 12), if $e$ separates $p$ - and $q$-region, and its endpoints are $p r q$ - and $q t p$-vertices.

Example (continued). We continue our running example. Let $v_{1}, v_{2}, e_{1}, e_{2}$, and $e_{3}$ be defined as in the left picture of Fig. 7. Then the vertex $v_{2}$ is an $a b \infty$-vertex and the vertex $v_{1}$ is a $b a \infty$-vertex. Edge $e_{1}$ is a $b a \infty a$-edge, $e_{2}$ is a $b \infty a \infty$-edge and $e_{3}$ is an $a b \infty b$-edge.

Lemma 3. Let $R \subseteq S$ and let $p, q, r$ and $t$ be sites in $R$. Then $V(R)$ contains at most one pqr-vertex and at most one prqt-edge.

Proof. We first show that there is at most one $p q r$-vertex. Assume otherwise that, say, $v$ and $w$ are two distinct $p q r$-vertices. Since $\operatorname{VR}(p, R)$ and $\operatorname{VR}(q, R)$ are path-connected, there are paths $\mathscr{P}$ and $\mathscr{Q}$ connecting $v$ and $w$ and running completely (except at their endpoints) inside $p$ - and $q$-regions, respectively. The cycle $\mathscr{P} \circ \mathscr{Q}$ then contains $r$-region in its interior and its exterior, a contradiction to the fact that $\operatorname{VR}(r, R)$ is homeomorphic to a disc. Thus there is at most one pqr-vertex.

The existence of two prqt-edges clearly contradicts the existence of at most one prq-vertex.
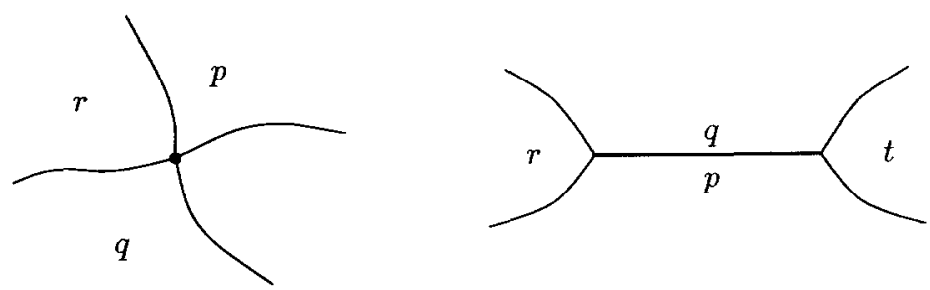

Fig. 12. A pqr-vertex and a prqt-edge. 
Four-tuples of sites not only allow us to distinguish between different edges of the same diagram, they furthermore capture all information necessary to compute the intersection of an edge with a new region.

Lemma 4 (basic operation lemma). Let e be a prqt-edge of $\mathrm{V}(R)$. Then the point set $e$ also constitutes a prqt-edge of $\mathrm{V}\left(R^{\prime}\right)$ for all $R^{\prime}$ with $\{p, r, q, t\} \subseteq R^{\prime} \subseteq R$. Moreover, $e \cap \overline{\operatorname{VR}(s, R \cup\{s\})}=e \cap \overline{\operatorname{VR}\left(s, R^{\prime} \cup\{s\}\right)}$ for any $s \notin R$.

Proof. Since $\operatorname{VR}\left(o, R^{\prime}\right) \supseteq \operatorname{VR}(o, R)$ for $o \in R^{\prime}$, the point set $e$ is incident to the Voronoi regions of $p, q, r$ and $t$ w.r.t. $R^{\prime}$, too. In particular the ordering of these Voronoi regions around $e$ does not change. Thus $e$ is a prqt-edge in $\mathrm{V}\left(R^{\prime}\right)$ as well. Let $s \in S-R$ be arbitrary. Observe first that $e \cap \overline{\operatorname{VR}(s, R \cup\{s\})} \subseteq e \cap$ $\overline{V R\left(s, R^{\prime} \cup\{s\}\right)}$ follows from $\operatorname{VR}(s, R \cup\{s\}) \subseteq \operatorname{VR}\left(s, R^{\prime} \cup\{s\}\right)$. For the converse, let $x \in e \cap \overline{\operatorname{VR}\left(s, R^{\prime} \cup\{s\}\right)}$ be arbitrary. Since $e$ is an edge of $\mathrm{V}(R)$ separating $p$ - and $q$-region with respect to $R$, there are arbitrarily small neighborhoods $U$ of $x$ such that $U-e \subseteq \operatorname{VR}(p, R) \cup \operatorname{VR}(q, R)$. Since $x \in$

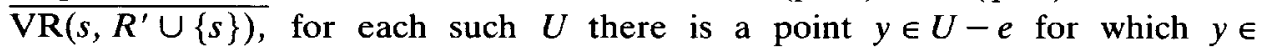
$\operatorname{VR}\left(s, R^{\prime} \cup\{s\}\right)$. On the other hand $y \in U-e$ implies $y \in \operatorname{VR}\left(p, R^{\prime}\right) \cup$ $\operatorname{VR}\left(q, R^{\prime}\right)$. We conclude $y \in D(s, p)$ or $y \in D(s, q)$. Since $y \in \operatorname{VR}(p, R) \cup$ $\operatorname{VR}(q, R)$ this implies $y \in \operatorname{VR}(s, R \cup\{s\})$. The claim $x \in \overline{\operatorname{VR}(s, R \cup\{s\}}$ follows because we can assume $U$ to be arbitrarily small.

By Lemmata 3 and 4 a prqt-edge $e$ of $\mathrm{V}(R)$ is also the unique prqt-edge of $\mathrm{V}\{p, r, q, t\})$ and the intersection between $e$ and the region of site $s$ is the same in $\mathrm{V}(R \cup\{s\})$ as in $\mathrm{V}(\{p, r, q, t, s\})$. We therefore define the following operation as the basic operation of our algorithm.

\section{Basic Operation}

Input: a five-tuple $(p, r, q, t, s)$ such that

(1) $V(\{p, r, q, t\})$ contains a prqt-edge $e$, and

(2) $s \notin\{p, r, q, t\}$.

Output: The combinatorial structure of $e \cap \overline{\operatorname{VR}(s,\{p, r, t, s\})}$, i.e., one of the following:

(1) intersection is empty

(2) intersection is non-empty and consists of a single component:

(a) $e$ itself

(b) a segment of $e$ adjacent to the prq-endpoint

(c) a segment of $e$ adjacent to the $q t p$-endpoint

(d) a segment not adjacent to any endpoint of $e$

(3) intersection is non-empty and consists of exactly two components.

Each call of basic_op will be charged one time unit. Note that the input to the basic operation is a combinatorial object, namely the 5-tuple $(p, r, q, t, s)$, and 
that the output is a combinatorial object, namely a symbol in $\{1,2 a, 2 b, 2 c, 2 d, 3\}$. Also note that the six cases specified exhaust all possible caes by Lemma 2 and that in case 3 the two components are incident to one endpoint of $e$ each. We use basic_op $(p, r, q, t, s)$ to denote the output of the basic operation on input $(p, r, q, t, s)$.

It is clear that the basic operation can also be used to decide whether an edge is intersected or clipped by a site. Let $e$ be a prqt-edge of $\mathrm{V}(R)$ and $s \in S-R$. Then $s$ intersects $e$ iff basic_op $(p, r, q, t, s) \in\{2 a, 2 b, 2 c, 2 d, 3\}$ and $s$ clips $e$ at the prq-endpoint iff basic_op $(p, r, q, t, s) \in\{2 a, 2 b, 3\}$.

Example (continued). In $\mathrm{V}(R), \quad R=\{a, b, \infty\}$, of Fig. 7 we have basic $\mathrm{op}(b, \infty, a, \infty, c)=2 b$, basic_op $(a, b, \infty, b, c)=2 c$ and basic_op $(b, a, \infty, a, c)=1$. Thus site $c$ intersects edges $e_{2}$ and $e_{3}$ and clips edges $e_{2}$ and $e_{3}$ at their endpoint $v_{2}$ with respect to $R=\{a, b, \infty\}$.

We have seen that four sites uniquely define an edge in the sense that there is no other edge defined by the same tuple of sites. However, an edge may in this way be defined by several different four-tuples of sites. In the analysis of our algorithm in Section 5 and for the presentation of the algorithm we need a stronger combinatorial characterization of edges.

Definition 5. (1) Let $e$ be an edge of $\mathrm{V}(R)$ separating $p$ - and $q$-region. Let $f_{p}$ and $g_{p}$ be the edges preceding and following $e$ in a clockwise traversal of the boundary of $\operatorname{VR}(p, R)$, and let $f_{q}$ and $g_{q}$ be the edges preceding and following $e$ in a counter-clockwise traversal of the boundary of $\operatorname{VR}(q, R)$, cf. Fig. 13. Assume further that $f_{p}$ separates $p$ - and $r_{p}$-region, $g_{p}$ separates $p$ - and $t_{p}$-region, and $g_{q}$ separates $q$ - and $t_{q}$-region, and $f_{q}$ separates $q$ - and $r_{q}$-region. Then $D_{R}(e)=$ $\left\{\left(r_{q}, q, p, r_{p}\right),\left(t_{p}, p, q, t_{q}\right)\right\}$ is called the description of $e$ with respect to $R$. By $\operatorname{set}\left(D_{R}(e)\right)$ we denote the set $\left\{p, q, r_{p}, r_{q}, t_{p}, t_{q}\right\}$.

(2) Let $D$ be the description of an edge $e$ of $\mathrm{V}(R)$, and let $s \in S-\operatorname{set}(D)$. Then site $s$ intersects description $D$ iff $e \cap \overline{\operatorname{VR}(s, \operatorname{set}(D) \cup\{s\})} \neq \emptyset$.

Example (continued). In $\mathrm{V}(R), R=\{a, b, \infty\}$, of Fig. 7 the edges have the following descriptions: $D_{R}\left(e_{1}\right)=\{(a, b, \infty, a),(a, \infty, b, a)\}, D_{R}\left(e_{2}\right)=\{(\infty, b, a, \infty)$, $(\infty, a, b, \infty)\}$, and $D_{R}\left(e_{3}\right)=\{(b, a, \infty, b),(b, \infty, a, b)\}$.

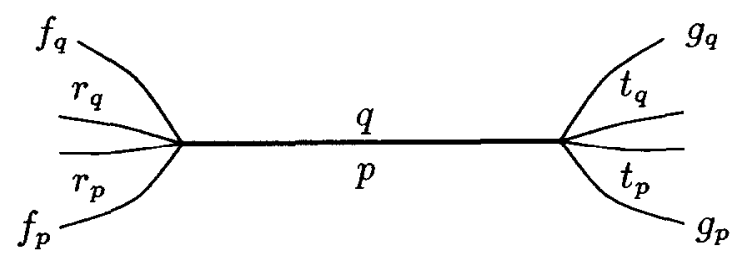

Fig. 13. An edge with description $\left\{\left(r_{q}, q, p, r_{p}\right),\left(t_{p}, p, q, t_{q}\right)\right\}$. 
Remarks. (1) The basic operation lemma has the following consequence: An edge $e$ of $\mathrm{V}(R)$ with description $D$ is also an edge of $\mathrm{V}(\operatorname{set}(D))$, and site $s \in S-R$ intersects edge $e$ with respect to $R$ iff $s$ intersects $e$ with respect to $\operatorname{set}(D)$ iff $s$ intersects $D$. Moreover, using our basic operation we can decide in constant time whether or not a site $s \in S-\operatorname{set}(D)$ intersects description $D$.

(2) In the case of general position, i.e., if Voronoi vertices have degree 3 , the four-tuple 'defining' an edge and the description of the edge contain the same set of sites. In fact, in that case, the whole analysis could also be done with four-tuples as descriptions. Descriptions are only introduced for the handling of degenerate cases, especially in the analysis of the algorithm in Section 5.

\section{The incremental algorithm}

In this section, we describe the incremental construction algorithm. The algorithm starts with the set $R_{3}=\{\infty, p, q\}$, where $p$ and $q$ are chosen uniformly at random from $S-\{\infty\}$, and then adds the remaining sites in random order, i.e., $R_{k+1}=R_{k} \cup\{s\}$, where $s$ is chosen uniformly at random from $S-R_{k}$. The following data structures are maintained for the current set $R=R_{k}$ of sites:

(1) The Voronoi diagram $\mathrm{V}(R)$ : It is stored as a planar map; with every face of $\mathrm{V}(R)$ the corresponding site in $R$ is stored.

(2) The history graph $\mathscr{l}(R)$ : It is a directed acyclic graph with a single source. Its vertex set is given by

$$
\{\text { source }\} \cup \underset{3 \leqslant i \leqslant k}{\bigcup}\left\{D_{R_{i}}(e) \mid e \text { is an edge of } \mathrm{V}\left(R_{i}\right)\right\} \text {. }
$$

The following history-graph invariants are maintained:

(1) Every vertex of $\mathscr{H}(R)$ has outdegree at most 5 and the vertices in $\left\{D_{R}(e) \mid e\right.$ edge of $\mathrm{V}(R)$ \} have outdegree 0 , i.e., are leaves of the graph.

(2) Every edge $e$ of $\mathrm{V}(R)$ is linked to its corresponding description $D_{R}(e)$ of $\mathscr{H}(R)$ and vice versa.

(3) For every site $s \in S-R$ and every leaf $D$ of $\mathscr{H}(R)$ that is intersected by $s$ there is a path from source to $D$ whose vertices are all interested by $s$.

We now discuss how to construct $\mathrm{V}(R \cup\{s\})$ and $\mathscr{H}(R \cup\{s\})$ from $\mathrm{V}(R)$ and $\mathscr{H}(R)$. To this aim let $E_{s}=\{e \mid e$ is an edge of $\mathrm{V}(R)$ and $e$ is intersected by $s\}$. We first show how to construct $E_{s}$ (Step 1), from $\mathscr{H}(R)$ and $\mathrm{V}(R)$ in time proportional to the number $c$ of vertices of $\mathscr{H}(R)$ which are intersected by $s$. Given $E_{s}$, it is then easy to construct $\mathrm{V}(R \cup\{s\}$ ) (Step 2), and $\mathscr{H}(R \cup\{s\}$ ) (Step 3) in time $\mathrm{O}\left(\left|E_{s}\right|\right)$.

Step 1: Construction of $\boldsymbol{E}_{s}$. Starting at the source of $\mathscr{H}(R)$ we explore all descriptions in $\mathscr{H}(R)$ which are intersected by $s$. Since the outdegree of $\mathscr{H}(R)$ is 
bounded by 5 , the number of visited vertices is proportional to $c$. Note that we can decide in constant time whether or not a description is intersected by a site by using our basic operation. Thus the search takes time $\mathrm{O}(c)$. Also, by the third history-graph invariant, it identifies all leaves of $\mathscr{t}(R)$ intersected by $s$. By the second history-graph invariant this set immediately gives the set of edges of $\mathrm{V}(R)$ whose descriptions are intersected by $s$. By the basic operation lemma this is set $E_{s}$. We conclude the following.

Lemma 5. The set $E_{s}$ can be computed in time $\mathrm{O}(c)$.

Step 2: Construction of $\mathbf{V}(R \cup\{s\})$. As above, let $\mathscr{S}=\operatorname{VR}(s, R \cup\{s\})$. We know from Lemma 1 that $\mathscr{S} \neq \emptyset$ iff $E_{s} \neq \emptyset$. So, $\mathrm{V}(R \cup\{s\})=\mathrm{V}(R)$ and $\mathscr{H}(R \cup$ $\{s\})=\mathscr{H}(R)$ if $E_{s}=\emptyset$. We therefore assume from now on that $E_{s} \neq \emptyset$. For an edge $e \in E_{s}, e-\overline{\mathscr{F}}$ consists of at most two subsegments of $e$. Also, if $e$ is a prqt-edge of $\mathrm{V}(R)$, basic_op $(p, r, q, t, s)$ tells us the structure of $e-\overline{\mathscr{T}}$. We call a point $v$ an endpoint of $e-\overline{\mathscr{S}}$ if it is an endpoint of one of the subsegments of $e$. In this way, $e-\overline{\mathscr{F}}$ may have 0,2 , or 4 endpoints. These endpoints are distinct by Lemma 2. We first characterize the vertices of $\mathrm{V}(R \cup\{s\})$. Let $V$ be the set of vertices of $\mathrm{V}(R)$ and let:

$V_{\text {del }}=\{v \mid v \in V$ and all edges incident to $v$ are clipped at $v$ by $s\}$,

$V_{\text {unch }}=\{v \mid v \in V$ and no edge incident to $v$ is clipped at $v$ by $s\}$,

$V_{\text {chang }}=\{v \mid v \in V$ and some but not all edges incident to $v$ are clipped at $v$ by $s\}$,

$V_{\text {new }}=\left\{v \mid v \notin V\right.$ and $v$ is endpoint of $e-\overline{\mathscr{T}}$ for some $\left.e \in E_{s}\right\}$.

Example (continued). Let $R=\{a, b, \infty\}$ and $s=c$. Then $V_{\mathrm{del}}=\emptyset, V_{\text {unch }}=\left\{v_{1}\right\}$, $V_{\text {chang }}=\left\{v_{2}\right\}$, and $V_{\text {new }}=\left\{v_{3}, v_{4}\right\}$. Note that our basic operation tells us that $e_{2}-\overline{\mathscr{S}}$ connects $v_{1}$ and $v_{3}$ and $e_{3}-\overline{\mathscr{Y}}$ connects $v_{1}$ and $v_{4}$, cf. Fig. 14 .

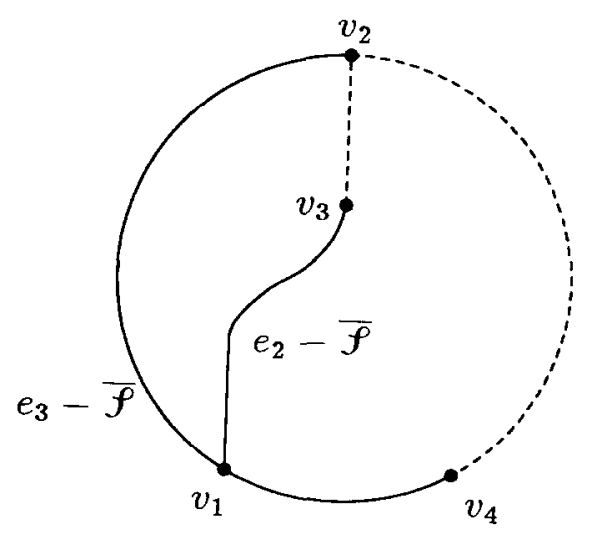

Fig. 14. $V(R) \cap \overline{\mathscr{S}}$ is shown dashed. 
Lemma 6. Every vertex $v$ of $\mathrm{V}(R \cup\{s\})$ is contained in $V_{\text {unch }} \cup V_{\text {chang }} \cap V_{\text {new }}$.

Proof. For every vertex $v$ of $\mathrm{V}(R \cup\{s\})$ there are two sites $p$ and $q$ different from $s$ such that an edge $e^{\prime}$ of $V(R \cup\{s\})$ separating $p$ - and $q$-region is incident to $v$. Also, there is an edge $e$ of $\mathrm{V}(R)$ with $e^{\prime} \subseteq e$. Thus $v$ is either a vertex of $\mathrm{V}(R)$ or $v$ lies on edge $e$ of $\mathrm{V}(R)$. In the latter case, $v$ is an endpoint of $e-\overline{\mathscr{S}}$ and hence $v \in V_{\text {new }}$. In the former case, $e$ is not clipped at $v$ by $s$ and hence $v \in V_{\text {unch }} \cup$ $V_{\text {chang. }}$.

Lemma 7. Let $v \in V_{\text {unch. Then }} U \cap \mathrm{V}(R)=U \cap \mathrm{V}(R \cup\{s\})$ for all sufficiently small neighborhoods $U$ of $v$; in particular, $v$ is a vertex of $\mathrm{V}(R \cup\{s\})$.

Proof. If $v \in V_{\text {unch }}$ then no edge of $\mathrm{V}(R)$ incident to $v$ is clipped at $v$ by $s$. Thus $U \cap \mathrm{V}(R) \cap \overline{\mathscr{S}} \subseteq v$ for all sufficiently small neighborhoods of $v$. Lemma 1 thus implies $U \cap \mathrm{V}(R) \cap \overline{\mathscr{F}}=\emptyset$ and hence $U \cap \mathrm{V}(R)=U \cap \mathrm{V}(R \cup\{s\})$.

Lemma 8. Let $v \in V_{\text {chang. }}$

(1) In the clockwise ordering of edges of $\mathrm{V}(R)$ around $v$, there are edges $f^{\prime \prime}$ and $f^{\prime}\left(f^{\prime}=f^{\prime \prime}\right.$ is possible) such that all edges between $f^{\prime \prime}$ and $f^{\prime}$ (inclusive) are not clipped at $v$ by $s$ and all edges between $f^{\prime}$ and $f^{\prime \prime}$ (exclusive) are clipped at $v$ by $s$.

(2) Let $e^{\prime}$ be the edge following $f^{\prime}$ and let $e^{\prime \prime}$ be the edge preceding $f^{\prime \prime}$ in the clockwise ordering of edges of $\mathrm{V}(R)$ around $v$, cf. Fig. 15. Let $f^{\prime}$ and $e^{\prime}$ border $p$-region and $e^{\prime \prime}$ and $f^{\prime \prime}$ border $q$-region. Then $v$ is a vertex of $\mathrm{V}(R \cup\{s\})$ incident to the following edges: all edges between $f^{\prime \prime}$ and $f^{\prime}$ (inclusive), an edge separating $p$ - and s-region, and an edge separating $s$ - and q-region.

Proof. (1) Since some but not all edges incident to $v$ are clipped at $v$ by $s, v$ must lie on bd $\mathscr{P}$. Since bd $\mathscr{P}$ is a simple closed curve passing through $v$, the edges clipped at $v$ by $s$ and the edges not clipped at $v$ by $s$ must form contiguous subsequences in the clockwise ordering of edges around $v$. This proves (1).

(2) This is an immediate consequence of part (1) and the piece of pie fact.

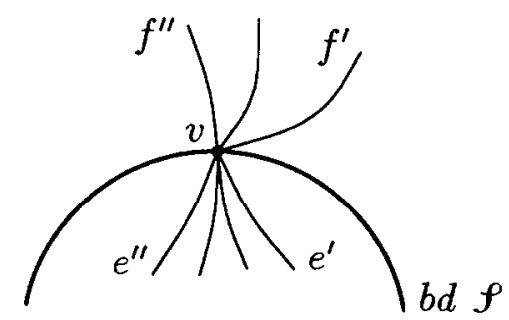

Fig. 15. A node $v \in V_{\text {chang. }}$ 

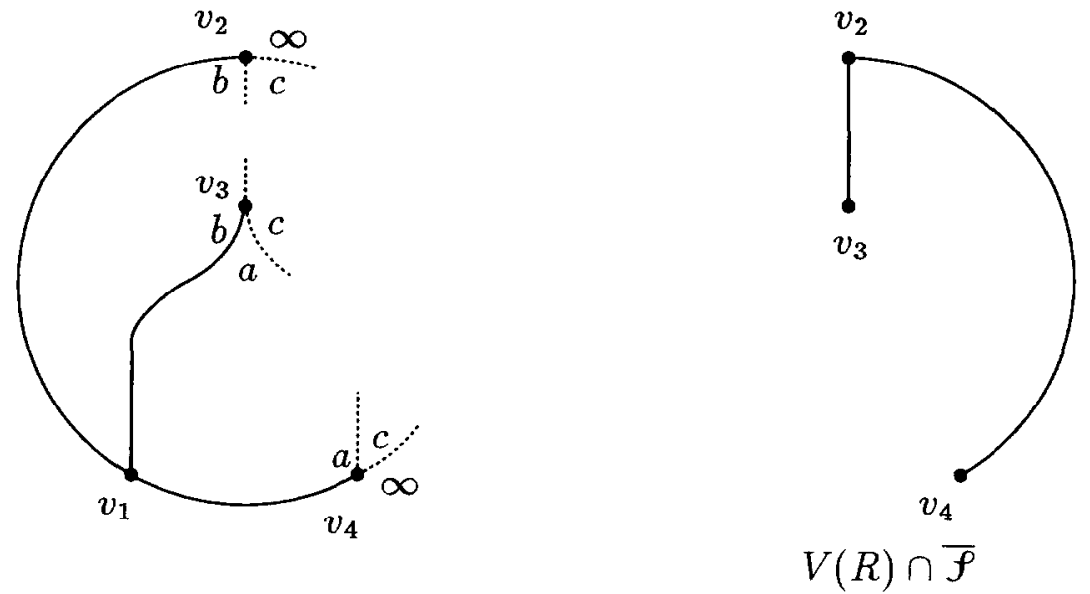

Fig. 16. On the left, for each vertex $v \in V_{\text {chang }} \cup V_{\text {new }}$ the two new edges incident to $v$ are indicated by dots. The deleted part of $V(R)$ is shown on the right.

Lemma 9. Let $v \in V_{\text {new }}$ and let $v$ lie on an edge $e$ of $V(R)$ separating $p$-and $q$-region. Then $v$ is a vertex of degree three in $\mathrm{V}(R \cup\{s\})$. The three edges incident

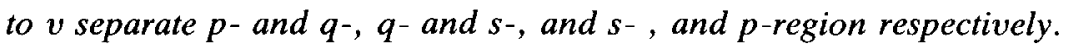

Proof. Obvious.

Example (continued). By Lemmas 6 to 9 , the vertices $v_{2}, v_{3}$ and $v_{4}$ become incident to two new edges each, cf. Fig. 16.

At this point, we have characterized the vertex set of $\mathrm{V}(R \cup\{s\})$ and also the set of edges incident to each vertex of $\mathrm{V}(R \cup\{s\})$ in their clockwise ordering around $v$. It remains to link the two occurrences of each edge. As above, let $\mathscr{E}=\mathrm{V}(R) \cap \overline{\mathscr{P}}$.

We know an embedding of $\mathscr{E}$ into the plane. The boundary of the outer face of $\mathscr{E}$ is a closed curve since $\mathscr{E}$ is connected. Also, the vertices on bd $\mathscr{F}$ lie on $\mathscr{E}$ and bd $\mathscr{S}$ is a simple closed curve. Hence a clockwise traversal of the boundary of the outer face of $\mathscr{E}$ yields the cyclic ordering of the 'half-edges' of bd $\mathscr{S}$, cf. Fig. 17. This allows us to link the two occurrences of each edge. We conclude the following.

Lemma 10. Given $E_{s}, \mathrm{~V}(R \cup\{s\})$ can be constructed from $\mathrm{V}(R)$ in time $\mathrm{O}\left(\left|E_{s}\right|\right)$.

Proof. Given $E_{s}$, one can determine the sets $V_{\text {del }}, V_{\text {chang, and }} V_{\text {new }}$ in time $\mathrm{O}\left(\left|E_{s}\right|\right)$. In the same time bound, one can update the cyclic adjacency lists of these vertices. Finally the traversal of $\mathscr{E}$ takes time $\mathrm{O}\left(\left|E_{s}\right|\right)$. 


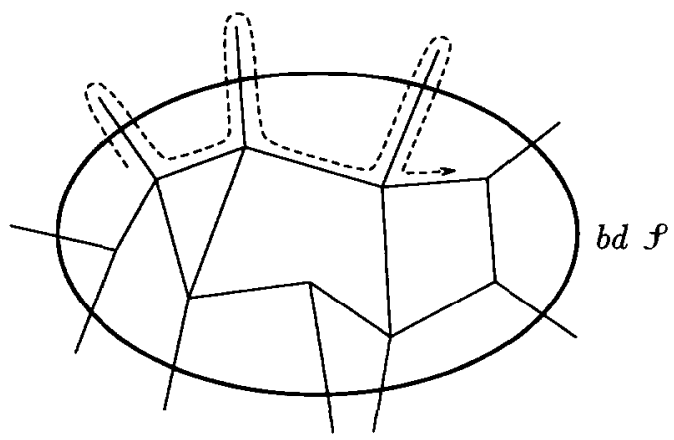

Fig. 17. Walking along the boundary of the outer face of $\mathscr{E}$.

Example (continued). The clockwise march around $\mathscr{E}$ and joining the two occurrences of each edge yields $\mathrm{V}(R \cup\{c\})$ as in Fig. 7 .

Step 3: Computation of $\mathscr{H}(\boldsymbol{R} \cup\{\boldsymbol{s}\})$. We first characterize the set of vertices $\mathscr{H}(R \cup\{s\})$ which are not already vertices of $\mathscr{H}(R)$. Call an edge $e$ of $\mathrm{V}(R \cup\{s\})$ new if it is not a subset of any edge of $\mathrm{V}(R)$, shortened if it is a proper subset of some edge of $\mathrm{V}(R)$, affected if $e$ is an edge of $\mathrm{V}(R)$ and there is a vertex $v \in V_{\text {chang }}$ such that $e$ is one of the edges $f^{\prime}$ or $f^{\prime \prime}$ defined in Lemma 8, cf. Fig. 18.

Lemma 11. Let $V_{\mathscr{H}}(R)$ and $V_{\mathscr{H}}(R \cup\{s\})$ be the vertex sets of $\mathscr{H}(R)$ and $\mathscr{H}(R \cup\{s\})$ respectively. Then $V_{\mathscr{H}}(R \cup\{s\})-V_{\mathscr{H}}(R)=\left\{D_{R \cup\{s\}}(e) \mid e\right.$ is a new, shortened, or affected edge of $\mathrm{V}(R \cup\{s\})\}$.

Proof. Let $e$ be an edge of $\mathrm{V}(R \cup\{s\})$ which is neither new, shortened, nor affected. Then $e$ has already been an edge of $\mathrm{V}(R)$ and hence its endpoints must lie in $V_{\text {unch }} \cup V_{\text {chang. }}$. Also, if an endpoint $v$ of $e$ belongs to $V_{\text {chang }}$ then $e$ lies strictly between the edges $f^{\prime \prime}$ and $f^{\prime}$ defined in Lemma 8. Thus $e$ 's descriptions with respect to $\mathrm{V}(R)$ and $\mathrm{V}(R \cup\{s\})$ are identical.

Conversely, if $e$ is new, shortened or affected, then $s$ contributes to $e$ 's description $D$ and therefore $D$ cannot be contained in $V_{\mathscr{K}}(R)$.

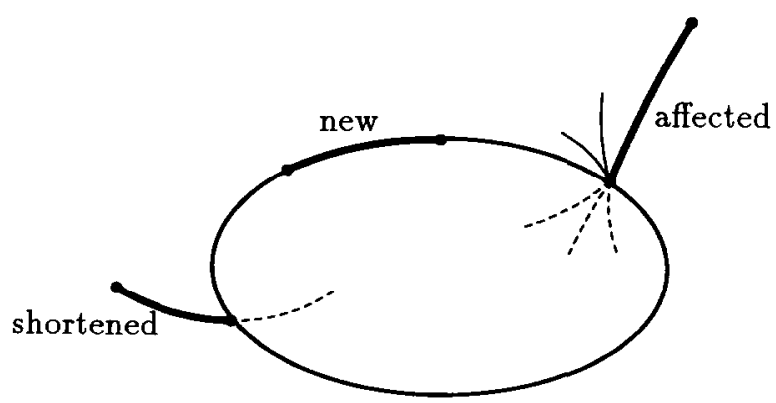

Fig. 18. A characterization of edges. 
We next discuss which edges have to be added to $\mathscr{H}(R \cup\{s\})$ in order to maintain the history-graph invariants.

Lemma 12. Let e be a shortened or affected edge of $\mathrm{V}(R \cup\{s\})$, let $e^{\prime}$ be the edge of $\mathrm{V}(R)$ with $e \subseteq e^{\prime}$, and let $t \in S-R-\{s\}$ intersect $e$ with respect to $R \cup\{s\}$. Then $t$ intersects $e^{\prime}$ with respect to $R$.

Proof. The lemma follows immediately from $e \cap \overline{\operatorname{VR}(t, R \cup\{s, t\})} \subseteq e^{\prime} \cap$ $\overline{\operatorname{VR}(t, R \cup\{t\})}$.

Thus for each shortened or affected edge $e$ we add the edge $\left(D_{R}\left(e^{\prime}\right), D_{R \cup\{s\}}(e)\right)$ to the history graph, where $e^{\prime}$ is the edge of $\mathrm{V}(R)$ with $e \subseteq e^{\prime}$.

For a new edge $e$ of $\mathrm{V}(R \cup\{s\})$ the situation is more complicated. We show that it is sufficient to make $e$ a child of all edges traversed during $e$ 's construction. To this end, let $x_{1}$ and $x_{2}$ be the endpoints of $e$, and let $p \in R$ be such that $e$ separates $p$ - and $s$-region in $\mathrm{V}(R \cup\{s\})$. By Lemma 1 there must be a path $\mathscr{P}$ in $\mathrm{V}(R) \cap \overline{\mathscr{S}}$ connecting $x_{1}$ and $x_{2}$. Without loss of generality we may assume that $\mathscr{P}$ is part of $\operatorname{bd} \operatorname{VR}(p, R) . \mathscr{P}$ is the part of $\mathrm{V}(R) \cap \overline{\mathscr{S}}$ traversed during the construction of $e$. Furthermore define the edges $e_{1}$ and $e_{2}$ of $\mathrm{V}(R)$ as follows. If $x_{1} \in V_{\text {new }}$, then let $e_{1}$ be the edge of bd $\operatorname{VR}(p, R)$ containing $x_{1}$. If $x_{1} \in V_{\text {chang }}$, then let $e_{1}$ be the edge of bdVR $(p, R)$ incident to $x_{1}$ and not contained in $\mathscr{P}$. The edge $e_{2}$ is defined analogously with respect to $x_{2}$. The reader may think of $e_{1}$ and $e_{2}$ as prolongations of $\mathscr{P}$ outside $\overline{\mathscr{P}}$. See Fig. 19 for an illustration of these definitions.

Lemma 13. Let e, $e_{1}, e_{2}$ and $\mathscr{P}$ be defined as above. Let $t \in S-R-\{s\}$ intersect $e$ w.r.t. $R \cup\{s\}$. Then there is an edge $g \in e_{1} \cup \mathscr{P} \cup e_{2}$ such that $t$ intersects $g$ with respect to $R$.

Proof. We assume for the sake of a contradiction that $t$ does not intersect any edge $g \in e_{1} \cup \mathscr{P} \cup e_{2}$. By the definition of $e_{1}$ and $e_{2}$ there are unique edges $e_{1}^{\prime}$ and $e_{2}^{\prime}$ of $\mathrm{V}(R \cup\{s\})$ such that $e_{1}^{\prime} \subseteq e_{1}$ and $e_{2}^{\prime} \subseteq e_{2}$.

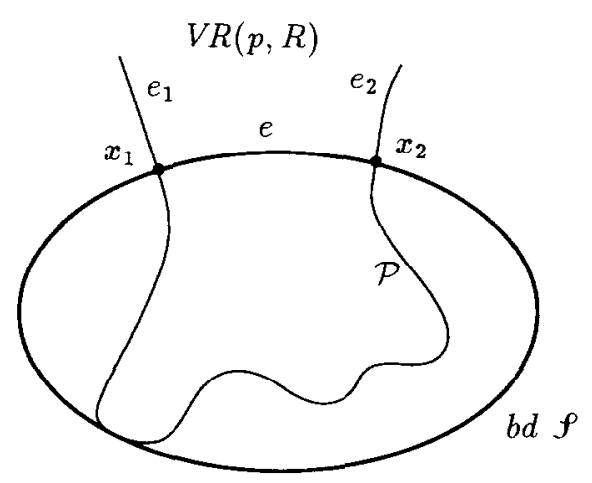

Fig. 19. The path $\mathscr{P}$ and edges $e_{1}$ and $e_{2}$. 
Claim 1. $t$ does not clip e, $e_{1}^{\prime}$ or $e_{2}^{\prime}$ at $x_{1}$ or $x_{2}$ with respect to $R \cup\{s\}$.

Proof. We first deal with edge $e$. Assume the contrary, say $t$ clips $e$ at $x_{1}$ w.r.t. $R \cup\{s\}$. Assume first that $x_{1} \in V_{\text {new. }}$. Then $e_{1}$ is the edge of $\mathrm{V}(R) \quad$ containing $\quad x_{1} \quad$ and $\quad x_{1} \in e_{1} \cap \overline{\mathrm{VR}(t, R \cup\{s, t\})} \subseteq e_{1} \cap \overline{\mathrm{VR}(t, R \cup\{t\})}$ and hence $t$ intersects $e_{1}$, a contradiction. Assume next that $x_{1} \in V_{\text {chang. Then }}$ $x_{1} \in \overline{\mathrm{VR}(t, R \cup\{s, t\})} \subseteq \overline{\mathrm{VR}(r, R \cup\{t\})}$. In $\mathrm{V}(R), t$ thus clips one of the two edges of bd $\operatorname{VR}(p, R)$ incident to $x_{1}$, because $\overline{V R(t, R \cup\{t\})}$ cannot contain an isolated point $x_{1}$, due to Lemma 1 . Since both edges belong to $e_{1} \cup \mathscr{P}$, we have a contradiction.

Since $e_{1}^{\prime} \subseteq e_{1}$ and $e_{2}^{\prime} \subseteq e_{2}$ and because $t$ does not intersect $e_{1}$ or $e_{2}$ by assumption, $t$ cannot clip $e_{1}^{\prime}$ or $e_{2}^{\prime}$.

Claim 2. $\mathscr{P} \subseteq \overline{\mathscr{S}}$ and there is a point $x \in e \cap \overline{\operatorname{VR}(t, R \cup\{s, t\})}$ which does not lie on $\mathscr{P}$.

Proof. $\mathscr{P} \subseteq \overline{\mathscr{P}}$ holds by definition. Since $t$ intersects $e$ but does not clip $e$ at $x_{1}$ or $x_{2}$, the intersection $e \cap \overline{\mathrm{VR}(t, R \cup\{s, t\})}$ is a non-empty subsegment of $e$ not extending to either endpoint of $e$. This subsegment must contain a point $x$ not in $\mathscr{P}$ since $t$ does not intersect any edge on path $\mathscr{P}$.

Now consider the wedge at $x_{1}$ formed by $e$ and $e_{1}^{\prime}$. According to the above claim, $t$ does not clip $e$ at $x_{1}$ in $\mathrm{V}(R \cup\{s\})$. Thus all points in the wedge belong to $\operatorname{VR}(p, R \cup\{s, t\})$. The same holds true for the wedge at $x_{2}$. Since $\operatorname{VR}(p, R \cup$ $\{s, t\})$ is connected, there is a path 2 from $x_{1}$ to $x_{2}$ running completely inside $\operatorname{VR}(p, R \cup\{s, t\}) \subseteq \operatorname{VR}(p, R \cup\{t\})$ expect at the endpoints, cf. Fig. 20. We may assume that $\mathscr{P}$. does not touch bd $\mathscr{P}$ (and therefore $x$ does not lie on 2). Thus $x$ lies in the interior of the cycle $x_{1} \circ \mathscr{P} \circ x_{2} \circ \mathscr{Q}$; otherwise $\operatorname{VR}(p, R)$ would not be simply connected. The point $x$ belongs to $\overline{V R(t, R \cup\{t\})}$. Since $\operatorname{VR}(p, R \cup\{t\})$ is simply connected, the region $\operatorname{VR}(t, R \cup\{t\})$ cannot be contained in the cycle $x_{1} \circ \mathscr{P} \circ x_{2} \circ 2$. Since $2 \cap \overline{\operatorname{VR}(t, R \cup\{t\})}=\emptyset$, we conclude $\mathscr{P} \cap \overline{\operatorname{VR}(t, R \cup\{t\})} \neq \emptyset$. The intersection cannot consists of a single point and hence $t$ must intersect an edge $g \in e_{1} \cup \mathscr{P} \cup e_{2}$, a contradiction. This completes the proof of Lemma 13.

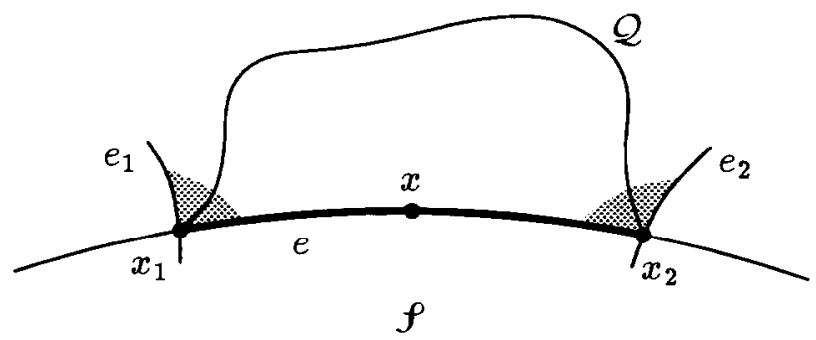

Fig. 20. The path 2 and the wedges at $x_{1}$ and $x_{2}$. 
In view of Lemma 13 we add edges $\left(D_{R}\left(e^{\prime}\right), D_{R \cup\{s\}}(e)\right)$ for any new edge $e$ of $\mathrm{V}(R \cup\{s\})$ and all $e^{\prime} \in e_{1} \cup \mathscr{P} \cup e_{2}$.

Lemma 14. The history-graph invariants hold for $\mathscr{H}(R \cup\{s\})$.

Proof. Part (3) of the history-graph invariant is maintained by Lemmas 12 and 13. Part (2) is trivial. For part (1) first observe that only leaves of $\mathscr{H}(R)$ can get new children. Thus the outdegree of inner nodes of $\mathscr{H}(R)$ does not change. We now show that a leaf of $\mathscr{H}(R)$ gets at most five children. We distinguish sevcral cases. Let $e^{\prime}$ be an edge of $\mathrm{V}(R)$. If $e^{\prime}$ is also an edge of $\mathrm{V}(R \cup\{s\})$ and $D_{R}\left(e^{\prime}\right)=D_{R \cup\{s\}}\left(e^{\prime}\right)$, then no edges out of $D_{R}\left(e^{\prime}\right)$ have been added to the history graph. Otherwise, either $e^{\prime} \subseteq \overline{\mathscr{S}}$ or there is a shortened or affected edge $e$ of $\mathrm{V}(R \cup\{s\})$ with $e \subseteq e^{\prime}$. In the former case, $e^{\prime}$ belongs to at most two paths $\mathscr{P}$. In the latter case, $e^{\prime}$ assumes the role of $e_{1}$ or $e_{2}$ at most four times and is also parent of $e$, i.e., the outdegree of $e^{\prime}$ is at most 5. It remains to prove that the desccriptions of edges in $V(R \cup\{s\})$ are leaves of $\mathscr{H}(R \cup\{s\})$. This follows from the fact that only those leaves of $\mathscr{H}(R)$ get children that are no longer descriptions of edges of $\mathrm{V}(R)$.

Fig. 21 shows a situation where the outdegree is actually 5 .

Lemma 15. Given $E_{s}, \mathscr{H}(R \cup\{s\})$ can be constructed from $\mathrm{V}(R)$ and $\mathscr{H}(R)$ in time $\mathrm{O}\left(\left|E_{s}\right|\right)$.

Proof. Follows from the discussion above.

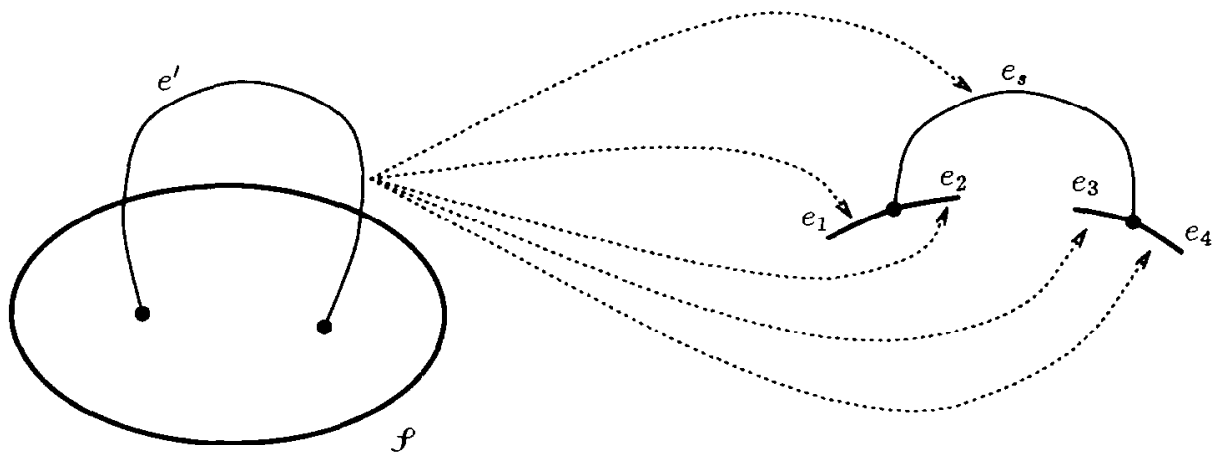

$V(R)$

$$
V(R \cup\{s\})
$$

Fig. 21. $D_{R}\left(e^{\prime}\right)$ has five children in the history graph. $e_{s} \subseteq e^{\prime}$ is a shortened edge. $e_{1}, \ldots, e_{4}$ are new edges on bd $\mathscr{S}$. Observe that $e_{2} \neq e_{3}$ is possible. 
We summarize the following.

Theorem 1. (a) Let $\infty \in R$ and $s \in S-R$. Then $\mathrm{V}(R \cup\{s\})$ and $\mathscr{H}(R \cup\{s\})$ can be constructed from $\mathrm{V}(R)$ and $\mathscr{H}(R)$ in time $\mathrm{O}(c)$ where $c$ is the number of vertices of $\mathscr{H}(R)$ intersected by $s$.

(b) For $R \subseteq S,|R|=3$ and $\infty \in R$, the data structures $V(R)$ and $\mathscr{H}(R)$ can be set up in time $\mathrm{O}(1)$.

Proof. (a) Comprises Lemmas 5, 10 and 15.

(b) The Voronoi diagram $\mathrm{V}(R)$ for three sites $\infty, p$ and $q$ has the structure shown in Fig. 22. The history graph $\mathscr{H}(\{\infty, p, q\})$ for these three sites simply consists of a node for the source and cach of the three edges of $V(\{\infty, p, q\})$. The descriptions of the 3 edges of $V(\{\infty, p, q\})$ are made children of the source. Both structures can certainly be set up in time $O(1)$.

\section{Analysis}

The analysis of randomized incremental algorithms is always done in terms of objects, regions and conflicts between them. In our case the objects are the sites and the regions are descriptions.

Definition 6. Let $R \subseteq S$.

(1) A description $D$ over $R$ is a set $\left\{\left(r_{q}, q, p, r_{p}\right),\left(t_{p}, p, q, t_{q}\right)\right\}$, where $\left\{p, q, r_{p}, r_{q}, t_{p}, t_{q}\right\} \subseteq R$, and $\mathrm{V}\left(\left\{p, q, r_{p}, r_{q}, t_{p}, t_{q}\right\}\right)$ contains a bounded edge with description $D$. $\mathscr{F}(R)$ denotes the set of all $\operatorname{descriptions}$ over $R$ and $\operatorname{set}(D)$ denotes the set $\left\{p, q, r_{p}, r_{q}, t_{p}, t_{q}\right\}$.

(2) Let $D$ be a description over $R$ and let $s \in S-\operatorname{set}(D)$ be a site. Site $s$ conflicts with $D$ iff there is no bounded edge in $\mathrm{V}(\operatorname{set}(D) \cup\{s\})$ with description $D$. Define $\mathscr{F}_{0}(R)=\{D \in \mathscr{F}(R) \mid D$ does not conflict with any site $s \in R-\operatorname{set}(D)\}$.

If a site $s$ intersects a description $D$, then it also conflicts with description $D$. The converse is not true. Namely, if in Fig. 13 a site $s$ clips any of the edges $g_{p}$, $g_{q}, f_{p}, f_{q}$ at their common endpoint with $e$, then $s$ conflicts with $D_{R}(e)$. The sole
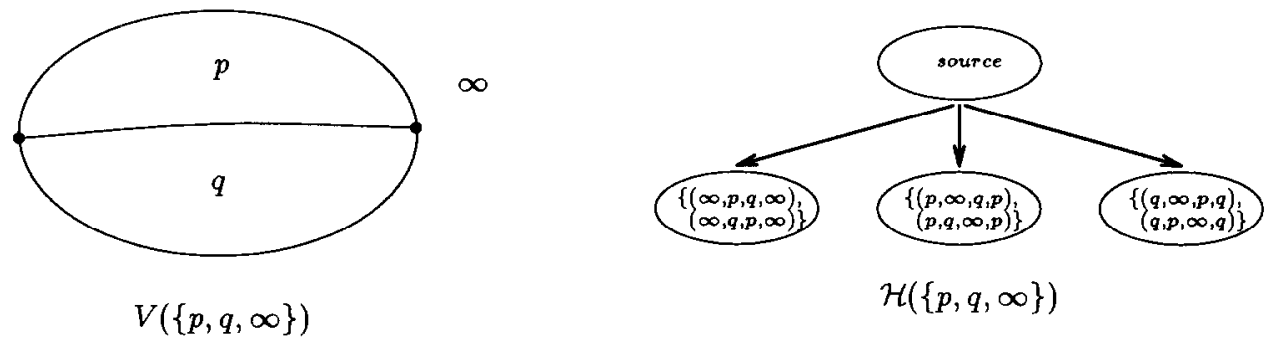

Fig. 22. Initialization of Voronoi diagram and history graph. 
motivation for defining the notion of conflict is the following bijections lemma. It makes the general results about randomized incremental constructions available for the analysis of our algorithm.

Lemma 16 (bijection lemma). Let $\infty \in R \subseteq S$. Then $e \mapsto D_{R}(e)$ is a bijection between the edges of $\mathrm{V}(R)$ and the descriptions in $\mathscr{F}_{0}(R)$.

Proof. Note first that all edges of $\mathrm{V}(R)$ are bounded since $\infty \in R$. Let $e$ be an edge of $\mathrm{V}(R)$, let $D=D_{R}(e)$, and let $s \in R-\operatorname{set}(D)$. By the basic operation lemma, $e$ is also an edge of $\mathrm{V}(\operatorname{set}(D))$ and $\mathrm{V}(\operatorname{set}(D) \cup\{s\})$. The same argument shows that the description of $e$ in $\mathrm{V}(\operatorname{set}(D))$ and $\mathrm{V}(\operatorname{set}(D) \cup\{s\})$ is still $D$. In particular $s$ does not conflict with $D$, i.e., $D \in \mathscr{F}_{0}(R)$. We have now shown that the mapping $e \mapsto D_{R}(e)$ maps the set of edges of $\mathrm{V}(R)$ into $\mathscr{F}_{0}(R)$. The mapping is injective since the existence of two different edges with the same description clearly contradicts Lemma 3 . It remains to show surjectivity.

Let $D \in \mathscr{F}_{0}(R)$ be arbitrary. We show that there is an edge in $\mathrm{V}(R)$ with description $D$. Assume the contrary. Then there are a set $R^{\prime}, \operatorname{set}(D) \subseteq R^{\prime} \subseteq R$, and a site $s \in R-R^{\prime}$ such that $\mathrm{V}\left(R^{\prime}\right)$ contains an edge $e$ with description $D$, but $\mathrm{V}\left(R^{\prime} \cup\{s\}\right)$ does not. Since $D \in \mathscr{F}_{0}(R)$ there is an edge with description $D$ in $V(\operatorname{set}(D))$ as well as in $\mathrm{V}(\operatorname{set}(D) \cup\{s\})$. By the basic operation lemma both edges are equal to edge $e$. We now consider $e$ with respect to $V\left(R^{\prime}\right)$ and distinguish several cases according to whether or not $s$ intersects $e$ w.r.t. $R^{\prime}$. Thus let $e \cap \overline{\mathrm{VR}\left(s, R^{\prime} \cup\{s\}\right)} \neq \emptyset$. By the basic operation lemma we have $e \cap$ $\overline{\mathrm{VR}(s, \operatorname{set}(D) \cup\{s\})} \neq \emptyset$ in contradiction to the claim that $e$ is edge of $\mathrm{V}(\operatorname{set}(D) \cup$ $\{s\})$. Thus let now $e \cap \overline{\operatorname{VR}\left(s, R^{\prime} \cup\{s\}\right)}=\emptyset$. Then $e$ is also edge of $V\left(R^{\prime} \cup\{s\}\right)$. But then the description of $e$ w.r.t. $R^{\prime} \cup\{s\}$ must be different from $D$, say $D^{\prime}$. By the basic operation lemma $e$ then also has the description $D^{\prime}$ in $\mathrm{V}(\operatorname{set}(D) \cup\{s\}$ ), a contradiction.

I et $s_{1}, s_{2}, \ldots, s_{n}$ be the sequence in which the algorithm processes the sites and let $R_{r}=\left\{s_{1}, s_{2}, \ldots, s_{r}\right\}$, for $1 \leqslant r \leqslant n$. The bijection lemma provides an alternative characterization of the vertex set of the history graph.

Lemma 17. The set of nodes of $\mathscr{H}\left(R_{r}\right)$ equals $\{$ source $\} \cup \bigcup_{3 \leqslant i \leqslant r} \mathscr{F}_{0}\left(R_{i}\right)$.

Proof. Obvious.

Lemma 17 characterizes the vertex set of the history graph as a set of combinatorial objects defined by a small number of input sites. We can therefore apply the results of $[8,4,7]$ to the analysis of our algorithm. To do so assume that the algorithm processes the sites in random order. $[8,4,7]$ give bounds on the expected size of the history graph and the number of its vertices in conflict with a input site in terms of $f_{r}$, the expected size of $\mathscr{F}_{0}(R)$ for a random subset $R \subseteq S$, $|R|=r$. 
Lemma 18 ([7], Theorems 3 and 4]). (1) The expected size of $\mathscr{H}\left(R_{r}\right)$ is $\mathrm{O}\left(\sum_{i \leqslant r} f_{i} / i\right)$.

(2) The expected number of vertices of $\mathscr{H}\left(R_{r-1}\right)$ in conflict with site $s_{r}$ is $\mathrm{O}\left(\sum_{i \leqslant r} f_{i} / i(i-1)\right)$.

Since a Voronoi diagram of $i$ sites has at most $3 i-6$ edges the bijection lemma implies $f_{i}=\mathrm{O}(i)$.

Theorem 2. An abstract Voronoi diagram of $n$ sites can be computed by a randomized algorithm in expected time $\mathrm{O}(n \log n)$ and expected space $\mathrm{O}(n)$. Moreover, the expected time for inserting the $r$-th object is $\mathrm{O}(\log r)$. Randomization here only concerns the order in which the sites are inserted.

Proof. At any time the size of the history graph clearly dominates the size of the Voronoi diagram. Thus the expected space used by the algorithm is $\mathrm{O}(n)$ by Lemma 18.

By Theorem 1 the time needed to insert the $r$-th site $s_{r}$ is proportional to the number $c_{r}$ of vertices in $\mathscr{H}\left(R_{r-1}\right)$ which are intersected by $s_{r}$. Since each intersection implies a conflict we have $c_{r}=\mathrm{O}(\log r)$ by Lemma 18 . This yields the claimed time bounds.

\section{Simple Voronoi diagrams}

In this section we introduce a subclass of abstract Voronoi diagrams for which the basic operation can be replaced by two operations each requiring only four sites as input.

Definition 7. A system of bisectors is called simple if for any three (finite) sites the induced Voronoi diagram contains at most one vertex.

Observe that in general three sites $p, q$ and $r$ can produce two vertices, a $p q r$ and a prq-vertex, see Fig. 3 for an example. Simple systems of bisectors are generated, for instance, by point sites under the Euclidean metric or under the $L_{1}$-metric (as defined by Lee [17]), and by Powerdiagrams (see [1]).

Now consider a Voronoi diagram $\mathrm{V}(R), \infty \in R \subseteq S$, generated by a simple system of bisectors. As in the previous sections let $s \in S-R$. We investigate again the type of intersection between an edge of $\mathrm{V}(R)$ and site $s$. For this investigation edges on $\Gamma$ must be treated separately.

To this purpose, let $e$ be a prqt-edge of $\mathrm{V}(R)$ not on $\Gamma$, i.e., $p, q \neq \infty$. Since $\mathrm{V}(\{p, q, s\})$ contains at most one vertex, cases $2 \mathrm{~d}$ and 3 of our basic operation are excluded. Furthermore cases $1,2 \mathrm{a}, 2 \mathrm{~b}$, and $2 \mathrm{c}$ can be distinguished simply by 

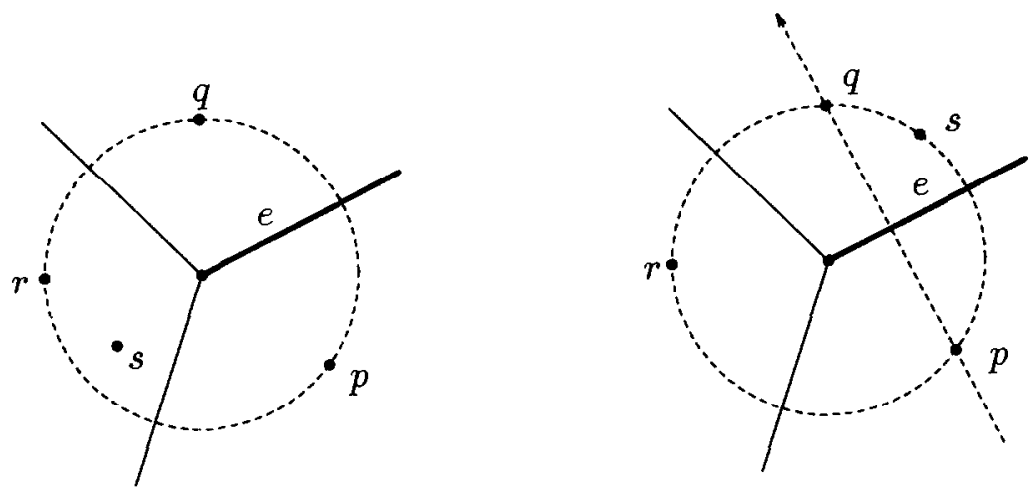

Fig. 23. $s$ clips $e$ at the prq-endpoint.

deciding whether $s$ clips $e$ at its prq-or qtp-endpoint (see Fig. 23).

$$
\text { basic_op }(p, r, q, t, s)= \begin{cases}1 & \text { iff } s \text { does not clip } e \text { at either endpoint, } \\ 2 a & \text { iff } s \text { clips } e \text { at both endpoints, } \\ 2 b & \text { iff } s \text { clips } e \text { only at the prq-endpoint, } \\ 2 c & \text { iff } s \text { clips } e \text { only at the qtp-endpoint }\end{cases}
$$

However, clipping can be decided by looking at only four sites.

Lemma 19. Let e be a prqt-edge of $\mathrm{V}(R)$ and let $s \in S-R$. Let $e^{\prime}$ be the unique edge of $\mathrm{V}(\{p, q, r\})$ incident to the prq-vertex of $\mathrm{V}(\{p, q, r\})$ and separating $p$-region from q-region. Then $e \subseteq e^{\prime}$ and s clips $e$ at the prq-endpoint w.r.t. $R$ iff $s$ clips $e^{\prime}$ at the prq-endpoint w.r.t. $\{p, q, r\}$.

Proof. First observe that by the basic operation lemma $e$ is also a prqt-edge of $\mathrm{V}(\{p, q, r, t\})$ and that $e \cap \overline{\operatorname{VR}(s,\{p, q, r, s, t\})}=e \cap \overline{\operatorname{VR}(s, R \cup\{s\})}$. By removing $t$ from $\{p, q, r, t\}$ edge $e$ cannot shrink, but possibly grow at its $q t p$-endpoint. Thus $e \subseteq e^{\prime}$. For the remaining claim note that $t$ has no influence on whether $s$ clips $e$ at its prq-endpoint or not.

Thus, for edges not on $\Gamma$ the basic operation on five sites is reduced to a four sites clipping operation.

Let us now turn to edges on $\Gamma$. For these edges cases $2 \mathrm{~d}$ and 3 of our basic operation are also possible. Thus the basic operation cannot be reduced to clipping as before. However, for all these edges, one of the four 'defining' sites is always $\infty$. Moreover, if $e$ is a prqt-edge on $\Gamma$ then either $p=\infty$ or $q=\infty$. Since each prqt-edge is also a qtpr-edge, we can assume $p=\infty$. Computing the outcome of the basic operation for a prqt-edge on $\Gamma$ can thus be handled by a special basic_op_ $\Gamma$ operation that inputs only the four sites $q, r, t$ and $s$.

To give an impression of the amount of programming hidden inside the basic operation, we sketch the implementation for a Voronoi diagram of points under 

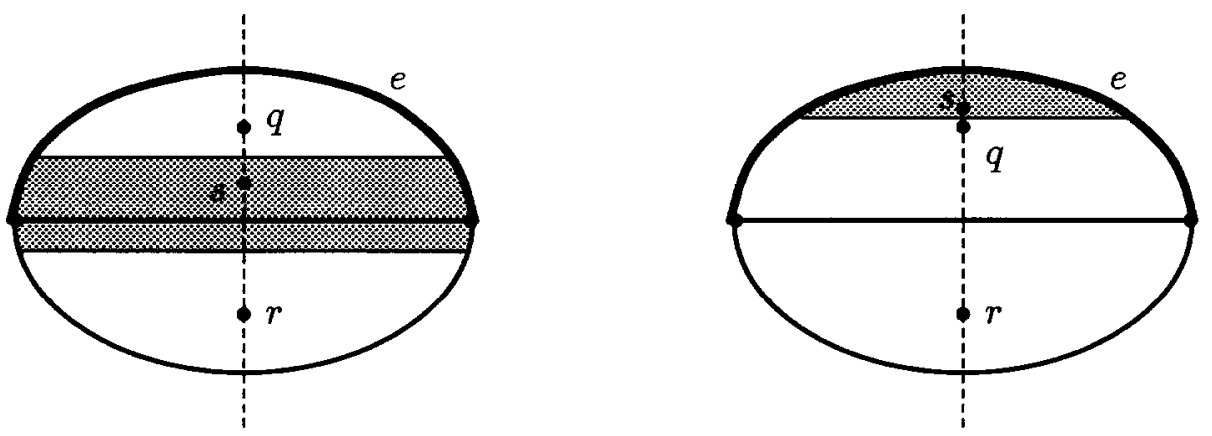

Fig. 24. Site $s$ intersects an edge $e$ on $\Gamma$.

the Euclidean metric. Let $e$ be a prqt-edge not on $\Gamma$, and furthermore let $r \neq \infty$. Then $s$ clips $e$ at its $p r q$-endpoint iff $s$ lies inside the circumcircle of $p, r$ and $q$, or $s$ lies on the circumcircle of $p, r$ and $q$, and $p, q$ and $s$ form a rightturn. If $r=\infty$ then $e$ is an 'unbounded' edge of $V(R-\{\infty\})$. The circumcircle of $p, r$ and $q$ then becomes the 'infinite circle' through $p, \infty$ and $q$, i.e., the line through $p$ and $q$; point $s$ lies inside the 'infinite circle' iff $s$ lies to the right of the line through $p$ and $q$ directed from $p$ to $q$. Furthermore, if $s$ lies on the line through $p$ and $q$ then $s$ clips iff $s$ lies between $p$ and $q$.

Let $e$ now be an orqt-edge on $\Gamma$. Cases $2 \mathrm{~d}$ and 3 of our basic operation can occur only if $r=t$ and $q, r$ and $s$ are collinear, see Fig. 24. If $r \neq t$ or $q, r$ and $s$ are not collinear then the outcome of the basic operation is once again completely determined by the way $s$ clips $e$ at its endpoints. Here $s$ clips $e$ at its $\infty r q$-endpoint iff $r, q$ and $s$ form a rightturn, or $s$ lies on the line through $r$ and $q$ between $r$ and $q$.

The test whether a point lies inside, on, or outside the circumcircle of three other points, and the test whether three points are collinear, or form a left- or rightturn are fundamental tests in computational geometry. Observe that all algorithms which use only the incircle-test do not handle four cocircular or three collinear points.

For Powerdiagrams the implementation is very similar, for diagrams of points under the $L_{1}$-metric it is more involved.

\section{Conclusion}

We have shown that the construction of abstract Voronoi diagrams can be reduced efficiently and purely combinatorially to the construction of abstract Voronoi diagrams for five sites, respectively four sites in some cases. This is also true for furthest site abstract Voronoi diagrams, see [20]. Many previously considered types of Voronoi diagrams can thus be handled by the same simple algorithm. 


\section{References}

[1] F. Aurenhammer, Power diagrams: Properties, algorithms and applications, SIAM J. Comput. 16 (1987) 78-96.

[2] F. Aurcnhammer, Voronoi diagrams-A survey of fundamental gcometric data structure, ACM Computing Surveys 23, (1991) 345-405.

[3] J.D. Boissonnat and M. Devillers-Teillaud, On the randomized construction of the Delaunay tree, Technical Report 1140, INRIA Sophia-Antipolis, 1989.

[4] J.D. Boissonnat, O. Devillers, R. Schott, M. Teillaud and M. Yvinec, Applications of random sampling to on-line algorithms in computational geometry, Discrete Comput. Geom. 8 (1992) $51-71$.

[5] J.D. Boissonnat, O. Devillers and M. Teillaud, An on-line construction of higher order Voronoi diagrams and its randomized analysis, in; J. Urrutia, ed., Proc. of the 2 nd Canadian Conference on Computational Geometry, Ottawa (1990) 278-281.

[6] K.Q. Brown, Voronoi diagrans from convex hulls, Inform. Process. Lell. 9 (1979) 223-228.

[7] K.L. Clarkson, K. Mehlhorn and R. Seidel, Four results on randomized incremental constructions, Technical Report MPI-I-92-112, Max-Planck-Institut für Informatik, 1992. A preliminary version was presented at the 9th Symposium on Theoretical Aspects of Computer Science (STACS '92), LNCS 577; 463-474.

[8] K.L. Clarkson and P.W. Shor, Applications of random sampling in computational geometry, II, Discrete Comput. Geom. 4 (1989) 387-421.

[9] H. Edelsbrunner and R. Seidel, Voronoi diagrams and arrangements, Discrete Comput. Geom. 1 (1986) 25-44.

[10] S. Fortune, A sweepline algorithm for Voronoi diagrams, Algorithmica 2 (1987) 153-174.

[11] L.J. Guibas, D.E. Knuth and M. Sharir, Randomized incremental construction of Delaunay and Voronoi diagrams, Algorithmica 7 (1992) 381-413.

[12] L.J. Guibas and J. Stolfi, Primitives for the manipulation of general subdivisions and the computation of Voronoi diagrams, ACM Trans. Graphics 4 (1985) 74-123.

[13] R. Klein, Abstract Voronoi diagrams and their applications (extended abstract). In $H$. Noltemeier, ed., Proc. of the Workshop on Computational Geometry and its Applications, Würzburg, LNCS 333 (1988) 148-157.

[14] R. Klein, Voronoi diagrams in the Moscow metric (extended abstract), in: J. van Leeuwen, ed., Proc. of the Workshop on Graphtheoretic Concepts in Computer Science, Amsterdam, LNCS 344 (1988) 434-441.

[15] R. Klein, Combinatorial properties of abstract Voronoi diagrams, in: M. Nagl, ed. Proc. of the Workshop on Graphtheoretic Concepts in Computer Science, Rolduc, LNCS 411 (1989) 356-369.

[16] R. Klcin, Concretc and Abstract Voronoi Diagrams, LNCS 400 (Springer, Berlin, 1989).

[17] D.T. Lee, Two-dimensional Voronoi diagrams in the $L_{p}$-metric. J. ACM 27 (1980) 604-618.

[18] D. Leven and M. Sharir, Intersection and proximity problems and Voronoi diagrams, in: J. Schwartz and C.K. Yap, eds., Advances in Robotics, Vol. 1 (Lawrence Erlbaum, London, 1986).

[19] K. Mehlhorn, S. Meiser and C. ÓDúnlaing, On the construction of abstract Voronoi diagrams, Discrete Comput. Geom. 6 (1991) 211-224.

[20] K. Mehlhorn, S. Meiser and R. Rasch, Furthest site abstract Voronoi diagrams, Technical Report MPI-I-92-135, Max-Planck-Institut für Informatik, 1992.

[21] M.I. Shamos and D. Hoey, Closet point problems, in: Proc. of the 16th Annual IEEE Symposium on Foundations of Computer Science (1975) 151-162.

[22] N. Zimmer, Die Konstruktion abstrakter Voronoidiagramme (in German), Master's Thesis, Universität des Saarlandes, 1992. 